\title{
Topological Nonsymmorphic Metals from Band Inversion
}

\author{
Lukas Muechler, ${ }^{1}$ A. Alexandradinata, ${ }^{2,3}$ Titus Neupert, ${ }^{4}$ and Roberto $\operatorname{Car}^{1,3}$ \\ ${ }^{1}$ Department of Chemistry, Princeton University, Princeton, New Jersey 08544, USA \\ ${ }^{2}$ Department of Physics, Yale University, New Haven, Connecticut 06520, USA \\ ${ }^{3}$ Department of Physics, Princeton University, Princeton, New Jersey 08544, USA \\ ${ }^{4}$ Princeton Center for Theoretical Science, Princeton University, Princeton, New Jersey 08544, USA \\ and Department of Physics, University of Zurich, Winterthurerstrasse 190, 8057 Zurich, Switzerland \\ (Received 14 May 2016; revised manuscript received 4 October 2016; published 29 December 2016)
}

\begin{abstract}
We expand the phase diagram of two-dimensional, nonsymmorphic crystals at integer fillings that do not guarantee gaplessness. In addition to the trivial, gapped phase that is expected, we find that band inversion leads to a class of topological, gapless phases. These topological phases are exemplified by the monolayers of $M \mathrm{Te}_{2}(M=\mathrm{W}, \mathrm{Mo})$ if spin-orbit coupling is neglected. We characterize the Dirac band touching of these topological metals by the Wilson loop of the non-Abelian Berry gauge field. Furthermore, we develop a criterion for the proximity of these topological metals to $2 \mathrm{D}$ and $3 \mathrm{D} \mathbb{Z}_{2}$ topological insulators when spinorbit coupling is included; our criterion is based on nonsymmorphic symmetry eigenvalues, and may be used to identify topological materials without inversion symmetry. An additional feature of the Dirac cone in monolayer $M \mathrm{Te}_{2}$ is that it tilts over in a Lifshitz transition to produce electron and hole pockets-a type-II Dirac cone. These pockets, together with the pseudospin structure of the Dirac electrons, suggest a unified, topological explanation for the recently reported, nonsaturating magnetoresistance in $\mathrm{WTe}_{2}$, as well as its circular dichroism in photoemission. We complement our analysis and first-principles band structure calculations with an ab-initio-derived tight-binding model for the $\mathrm{WTe}_{2}$ monolayer.
\end{abstract}

DOI: 10.1103/PhysRevX.6.041069

\section{INTRODUCTION}

In the Landau-Ginzburg paradigm [1], different phases of matter are distinguished by their symmetry. A recent major advance led to the recognition that insulators with the same symmetries and particle numbers can be topologically distinct [2-4]. That is, for the same integer electron filling and symmetry class, one may have either trivial or topological insulators. The latter have unusual electronic properties that originate [5] from the Berry phase [2,6] of electronic wave functions. This scenario is substantially modified for a broad class of crystals having nonsymmorphic symmetries, namely, the spatial symmetries that unavoidably translate the spatial origin by a fractional lattice vector [7]. Nonsymmorphic symmetries guarantee that at certain integer fillings the phase of matter must always be gapless. This robust and unavoidable metallicity originates from the nontrivial connectivity $[8,9]$ of elementary energy bands [10].

Our work explores the distinct phases of nonsymmorphic matter, but for integer fillings that do not guarantee gaplessness, as illustrated in Figs. 1(c)-1(d). We find that

Published by the American Physical Society under the terms of the Creative Commons Attribution 3.0 License. Further distribution of this work must maintain attribution to the author(s) and the published article's title, journal citation, and DOI.
Subject Areas: Condensed Matter Physics, Topological Insulators

a band inversion separates a trivial, gapped phase from a topological, gapless phase. The latter metal is concretely exemplified by $M \mathrm{Te}_{2}(M=\mathrm{W}, \mathrm{Mo})$ monolayers as model systems if spin-orbit coupling (SOC) is neglected, as we substantiate with $a b$ initio calculations and tight-binding models. Another example of a nonsymmorphic metal due to band inversion has been proposed for the hypothetical haeckelite structures of transition metal dichalcogenides [11-13].

We characterize this metal by a topological invariant based on the Wilson loops of the non-Abelian Berry gauge field, which contrasts with previous Abelian Berry-phase characterizations of topological metals [14]. Wilson loops not only fundamentally characterize band topology through holonomy [15], they have recently emerged as an efficient method to diagnose topological insulators from firstprinciples calculations [15-19]. We propose here that the Wilson loop is also a powerful tool to identify and characterize topological metals.

The low-energy excitations of $M \mathrm{Te}_{2}$ monolayers are described by Dirac fermions that are topologically distinct from the rotationally symmetric Dirac fermions in graphene. Precisely, the Dirac cone disperses so anisotropically that it "tilts over" in a Lifshitz transition, i.e., part of the upper Dirac cone dips below the nodal energy $(\epsilon=0)$, as illustrated in Fig. 1(a), resulting in a discontinuous change in the band contours at the nodal energy. We refer to 


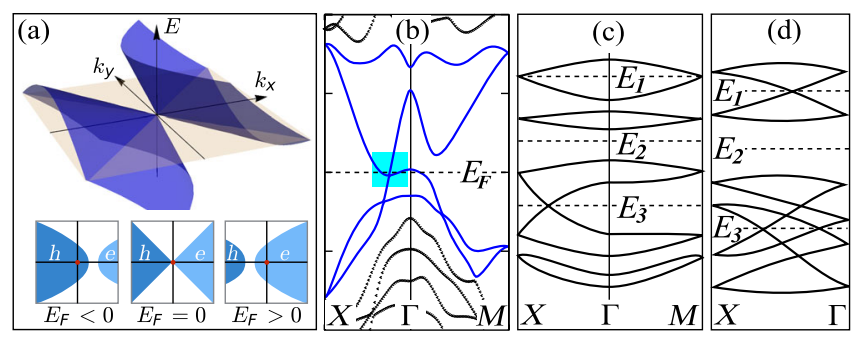

FIG. 1. (a) A Dirac fermion that tilts over. (b) Band structure of a $\mathrm{WTe}_{2}$ monolayer without spin-orbit coupling; the tilted Dirac fermion is highlighted by a blue square. (c) Band structure of a nonsymmorphic, spin-orbit-free crystal at various fillings. (d) Band structure of a nonsymmorphic, spin-orbit-coupled crystal at various fillings. For both (c) and (d), $E_{1}, E_{2}, E_{3}$ are Fermi energies that, respectively, exemplify (1) a semimetal whose metallicity originates from its filling, (2) a generic insulator, and (3) a topological semimetal whose metallicity originates from a band inversion.

it as a type-II Dirac cone, in analogy with a notion recently introduced for Weyl semimetals [20] that is, incidentally, materialized by 3D $M \mathrm{Te}_{2}$ [20-23]. Instead of a pointlike Fermi surface where the Fermi energy lies at a (potentially tilted) Dirac node [24-31], a type-II Dirac cone is characterized by electronlike and holelike Fermi surfaces that touch at the Dirac node [32] [see Fig. 1(a)]. This novel scenario promises an abundance of unexplored experimental possibilities, such as the observation of magnetic breakdown in transport experiments [33]. Our theory and tight-binding models should serve as important resources for ongoing experimental efforts [34,35] focused on the synthesis and the study of $M \mathrm{Te}_{2}$ monolayers.

The type-II Dirac fermions in spin-orbit-free $\mathrm{WTe}_{2}$ monolayers provide a unifying explanation for many phenomena in spin-orbit-coupled monolayers, bilayers, and $3 \mathrm{D}$ layered $\mathrm{WTe}_{2}$, where the spin-orbit coupling is weak $(\sim 0.1 \mathrm{eV})$ compared to the typical tight-binding hopping parameter $(\sim 1.3 \mathrm{eV})$. The latter are materials that have been fabricated and are under intense experimental scrutiny because of their giant, nonsaturating transverse magnetoresistance (MR) of $13 \times 10^{6} \%$ at $0.53 \mathrm{~K}$ and $60 \mathrm{~T}$, with new crystals achieving $1.7 \times 10^{6} \%$ at $2 \mathrm{~K}$ and $9 \mathrm{~T}$ [36,37]. Moreover, the angle-resolved photoemission (ARPES) of $\mathrm{WTe}_{2}$ exhibits circular dichroism (CD) [38], a phenomenon that typifies Dirac semimetals such as graphene $[39,40]$. We summarize our results as follows.

(i) When spin-orbit coupling is introduced, the degeneracy of the Dirac points is lifted and the disconnected bands are then topologically nontrivial [41] in the timereversal-symmetric $\mathbb{Z}_{2}$ classification $[4,42,43]$. In this work, we propose a criterion on the spin-orbit-free metal that is equivalently expressed by the number of Dirac fermions or the eigenvalues of the nonsymmorphic symmetry. If this criterion is satisfied, as is the case for $\mathrm{WTe}_{2}$, spin-orbit coupling induces $\mathbb{Z}_{2}$ topological order; beyond our case study of $\mathrm{WTe}_{2}$, our criterion may be used to identify other 2D and 3D topological materials without inversion symmetry. We remark that spin-orbit-coupled $\mathrm{WTe}_{2}$ remains metallic due to the persistence of its electron and hole pockets, which, again, originate from the tilted Dirac fermion.

(ii) In bilayer $\mathrm{WTe}_{2}$, the coupling between the two stacked monolayers breaks the nonsymmorphic symmetry that protects the Dirac fermions. The low-energy theory is then described by tilted Dirac fermions with small masses; the two-component wave function at each Fermi circle forms a pseudospin that rotates around the Dirac node, where Berry curvature [6] is concentrated.

(iii) The electron and hole pockets, Berry curvature, and rotating pseudospin are retained in $3 \mathrm{D} \mathrm{WTe}_{2}$, which comprises weakly coupled bilayers. We propose that the high mobilities in transport experiments should be attributed to suppressed backscattering due to the rotating pseudospin, while the observed circular dichroism should be correlated with the Berry phase of the Dirac cones $[39,40]$.

This work is organized as follows. After a preliminary description of the nonsymmorphic symmetries of $M \mathrm{Te}_{2}$ in Sec. II, we introduce the theory of band-inverted topological metals in Sec. III. In Sec. IV, we exemplify our theory with monolayer $\mathrm{WTe}_{2}$, for which we present a tightbinding model and introduce the notion of type-II Dirac cones that tilt over. We then extend our discussion to bilayer $\mathrm{WTe}_{2}$ in Sec. V, with focus on its dichroism. In Sec. VI, we summarize our results and further relate them to the magnetoresistance measurements in $3 \mathrm{D} \mathrm{WTe} \mathrm{W}_{2}$. Details on the derivation of the topological invariant, the role of spinorbit coupling, the tight-binding model, and the CD calculation are presented in Appendixes A, B, C, and D.

\section{NONSYMMORPHIC SPACE GROUPS AND RELEVANT CRYSTAL STRUCTURES}

In crystals, a basic geometric property that distinguishes spatial symmetries concerns how they transform the spatial origin: rotations, inversions, and reflections preserve the origin, while screw rotations and glide reflections unavoidably translate the origin by a rational fraction of the lattice period [7]. If no origin exists that is simultaneously preserved, modulo integer lattice translations, by all the symmetries in a space group, this space group is called nonsymmorphic. In Sec. II A, we exemplify a nonsymmorphic space group with the crystal structure of $M X_{2}$ monolayers, which applies to $\mathrm{WTe}_{2}, \mathrm{MoTe}_{2}$, and $\mathrm{ZrI}_{2}$. In contrast, the $M X_{2}$ bilayer is characterized by a symmorphic space group, as we explain in Sec. II B.

\section{A. Crystal structure of the $M X_{2}$ monolayer}

The $M$ atoms form zigzag chains along $\boldsymbol{e}_{x}$, and are coordinated by $X$ atoms that form distorted edge-sharing 
octahedra; here, $\boldsymbol{e}_{x}, \boldsymbol{e}_{y}, \boldsymbol{e}_{z}$ are basis vectors in a Cartesian coordinate system, with $\boldsymbol{e}_{z}$ orthogonal to the monolayer and $\boldsymbol{e}_{x}, \boldsymbol{e}_{y}$ the generators of the Bravais lattice of the monolayer; the lengths of $\boldsymbol{e}_{x}, \boldsymbol{e}_{y}$ correspond to lattice constants that we denote, respectively, by $a, b$. We label a unit cell in the monolayer by $\boldsymbol{R}=h \boldsymbol{e}_{x}+k \boldsymbol{e}_{y}$, with $h, k \in \mathbb{Z}$.

The group of a $M X_{2}$ monolayer is generated by (i) time reversal (T), (ii) lattice translations $t\left(\boldsymbol{e}_{x}\right)$ and $t\left(\boldsymbol{e}_{y}\right)$, where $t(\boldsymbol{r})$ indicates a translation by the vector $\boldsymbol{r} \in \mathbb{R}^{3}$, as well as (iii) a reflection $\bar{M}_{x} \equiv t\left(\boldsymbol{e}_{x} / 2\right) M_{x}$, which is a product of a reflection $M_{x}$, acting as $M_{x}:(x, y, z) \rightarrow(-x, y, z)$, and a translation $t\left(\boldsymbol{e}_{x} / 2\right)$ by half a lattice vector, and (iv) a screw rotation $\bar{C}_{2 x}=t\left(\boldsymbol{e}_{x} / 2\right) C_{2 x}$, which is the product of a twofold rotation $C_{2 x}$, acting as $C_{2 x}:(x, y, z) \rightarrow(x,-y,-z)$, and the same fractional translation. The product of the last two generators is the spatial inversion $\bar{M}_{x} \bar{C}_{2 x}=\mathcal{I}$ that sends $\boldsymbol{r} \rightarrow-\boldsymbol{r}$; we choose the inversion center, indicated by a green cross in Fig. 2(a), as our spatial origin. The atomic positions of the freestanding monolayers that we consider are extracted from monolayers within 3D crystals, whose parameters are known from experiments [44-46].

\section{B. Crystal structure of the $M X_{2}$ bilayer}

Let the position of each atom in a $M X_{2}$ monolayer be parametrized by $(x, y, z)$ relative to our chosen spatial origin [green cross Fig. 2(a)]. There is a corresponding identical atom positioned at $\left(-x+\frac{1}{2}+a,-y+b, z+c\right)$ in the second layer of the bilayer, where $\{a, b, c\}$ are

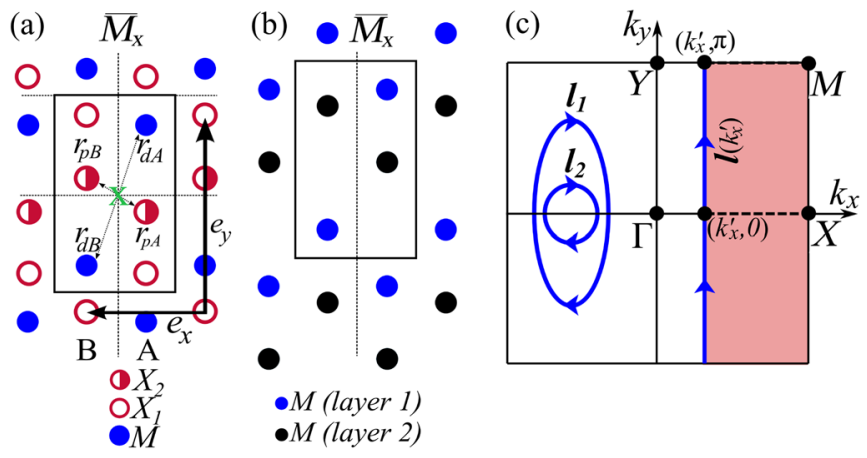

FIG. 2. (a) Crystal structure of a $M X_{2}$ monolayer. The horizontal lines are invariant under the screw $\bar{C}_{2 x}$, the vertical line is invariant under $\bar{M}_{x}$, and the green cross indicates the center of inversion and also our choice of spatial origin. Within each unit cell (encircled by the rectangle), we divide the four $X$ atoms into two pairs marked $X-1$ and $X-2$. The $X-2$ atoms are further divided into two sublattices labeled by A and B. $\boldsymbol{r}_{\ell, s}$, with $\ell \in\{d, p\}$ and $s \in\{A, B\}$, are vectors connecting the origin to the centers of the Wannier functions that we introduce in Sec. IVA. (b) Crystal structure of a $M X_{2}$ bilayer, where only the $M$ atoms are shown. (c) Brillouin zone of both the monolayer and the bilayer. In Sec. III, we characterize the monolayer by screw-symmetric Wilson loops indicated in blue: $l_{1}$ and $l_{2}$ are contractible, while $l\left(k_{x}^{\prime}\right)$ is not. material-specific parameters. This stacking spoils both inversion and screw symmetries of the monolayer, but retains the mirror symmetry $\bar{M}_{x}$, as we illustrate in Fig. 2(b). Our stacking is identical to that of the bilayer within 3D $M X_{2}$, whose experimentally known atomic positions [44] we use throughout this paper.

\section{THEORY OF BAND-INVERTED TOPOLOGICAL METALS}

In Sec. III A, we briefly review nonsymmorphic metals which are metallic only due to their filling. These metals are distinguished from metals that originate from band inversion, as we briefly describe in Sec. III A, and then more carefully elaborate in Sec. III B. These band-inverted metals admit a topological classification that we describe in Sec. III C. When a gap is induced by spin-orbit coupling, certain band-inverted metals turn into topological insulators, as we substantiate in Sec. III D.

\section{A. Comparing topological and filling-enforced metals}

We introduce two types of nonsymmorphic metals: (i) filling-enforced metals, which are guaranteed to be metallic at certain fillings determined by the space group [8], and (ii) topological metals, which are not guaranteed in the sense of (i), but are metallic due to a topological band inversion that we describe below. To exemplify (i) and (ii), we offer two examples from a group generated by $\left\{T, t\left(\boldsymbol{e}_{x}\right), t\left(\boldsymbol{e}_{y}\right), \bar{C}_{2 x}\right\}$, which has one less generator $\left(\bar{M}_{x}\right)$ than the group of $\mathrm{WTe}_{2}$; we define this smaller group to emphasize the relevant symmetries, as well as their wider applicability to other materials.

In this section, we consider electronic systems without spin-orbit coupling. In our definition of filling $(f)$, we count a spin-degenerate band as a single band, and each spin species transforms in an integer-spin representation $[7,47]$ of the space-group symmetries described in Sec. II; e.g., $\bar{C}_{2 x}^{2}=t\left(\boldsymbol{e}_{x}\right)$ would not include a $2 \pi$ rotation of the spin. There are two lines $\left(k_{y}=0\right.$ and $\left.\pi\right)$ that are individually mapped onto themselves under the screw transformation; in short, we call them screw lines. Bands along each screw line may be labeled by the eigenvalues of $\bar{C}_{2 x}$, which fall into the two momentum-dependent branches $\pm \exp \left(-i k_{x} / 2\right)$, as follows from $\bar{C}_{2 x}^{2}=t\left(\boldsymbol{e}_{x}\right)=$ $\exp \left(-i k_{x}\right)$ in a Bloch-wave representation. At inversioninvariant points $X\left[\left(k_{x}, k_{y}\right)=(\pi, 0)\right]$ and $M\left[\left(k_{x}, k_{y}\right)=\right.$ $(\pi, \pi)]$ on the screw lines, time reversal pairs up complexconjugate representations of $\bar{C}_{2 x}$, such that the bands are all doubly degenerate. Each degenerate subspace is composed of an equal number of states with $\bar{C}_{2 x}$ eigenvalue $+i$ and $-i$. In contrast, time reversal does not enhance the degeneracy at $\Gamma\left[\left(k_{x}, k_{y}\right)=(0,0)\right]$ and $Y\left[\left(k_{x}, k_{y}\right)=(0, \pi)\right]$, where the $\bar{C}_{2 x}$ eigenvalues are real. The situation is illustrated in Fig. 1(c): bands divide minimally into pairs, such that 
within each pair there is at least one robust contact point (here, a crossing between orthogonal screw representations at $X / M$ ) that connects both members of the pair-we say that bands are twofold connected along both screw lines [48]. The notion of connectivity of a submanifold (here, a screw line) generalizes [48] the notion of symmetryenforced degeneracy at an isolated wave vector; the connectivity of the entire Brillouin zone [8] relates to the theory of elementary energy bands [10]. Because of the twofold connectivity, any odd, single-spin filling $(f \in 2 \mathbb{Z}+1)$ is guaranteed to produce a filling-enforced metal, as exemplified by the Fermi energy $E_{1}$ in Fig. 1(c).

If the filling is even $(f \in 2 \mathbb{Z})$, metallicity is not guaranteed by reason of filling, as exemplified by the Fermi energy $E_{2}$ in Fig. 1(c). Dirac metallicity is, nevertheless, guaranteed at even filling due to independent band inversions along either screw line, e.g., a single inversion at $\Gamma$ (Y) would nucleate a pair of time-reversal-related Dirac crossings that situate anywhere along $X \Gamma X$ (along $M Y M$ ), as we illustrate with the spin-orbit-free $\mathrm{WTe}_{2}$ monolayer as a model system in Fig. 1(b). The general theory of band-inverted metals is elaborated in the next section. We remark that a glide reflection $\bar{M}_{y}$, composed of a reflection $(y \rightarrow-y)$ and a half-lattice translation in $\boldsymbol{e}_{x}$, also satisfies $\bar{M}_{y}^{2}=t\left(\boldsymbol{e}_{x}\right)$, just like $\bar{C}_{2 x}$. Consequently, every result in this and the next two sections applies also to $\bar{M}_{y}$, with the cosmetic substitution of the term "screw" with "glide."

\section{B. Band inversion and Dirac metallicity at even filling}

In this section, we focus on even filling and quantify the relation between band inversion and Dirac metallicity. We say bands are inverted if the filled states, at any wave vector along a screw line, transform nontrivially under the screw rotation. To further clarify "filled states at a wave vector" for a filling $f$, we say that a state at a wave vector is filled if it belongs to the lowest set of $f$ bands at that wave vector. Filled states, according to our unconventional notion of filling, often coincide with actual states below the Fermi level [e.g., $\mathrm{ZrI}_{2}$ in Fig. 3(a)]; exceptions include the hole or electron pockets of $\mathrm{WTe}_{2}$ and $\mathrm{MoTe}_{2}$ [Figs. 3(b) and 3(c)], which originate from the Dirac fermion tilting over, as we elaborate in Sec. IV C. Our notion of filled states more naturally generalizes to bosonic systems, as we elaborate. Furthermore, we now demonstrate how the symmetry analysis of filled states is predictive of the number of Dirac crossings, whether or not they tilt over.

Precisely, we would count, for any $\boldsymbol{k}$ along $X Г X(M Y M)$, the number of Dirac crossings along the screw line that connects $\boldsymbol{k}$ to $X(M)$ in the direction of increasing $k_{x}$, as indicated by the bottom (top) dashed line in Fig. 2(c). Henceforth, we refer to this number as the Dirac count $\left(D_{k}\right)$; e.g., $D_{\Gamma}$ is the number of crossings along half the screw line: $\Gamma X$. For illustration, we plot $D_{\left(k_{x}, 0\right)}$ for five case

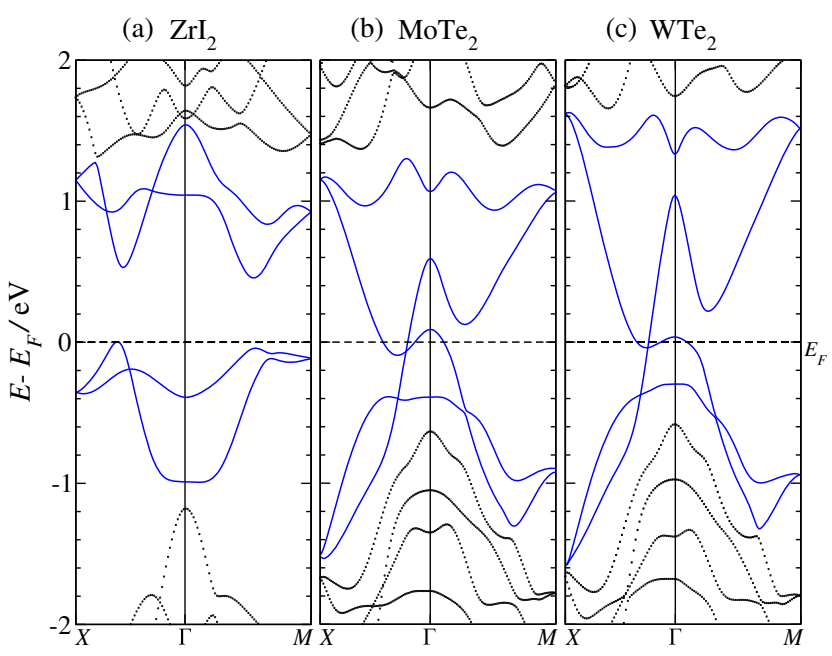

FIG. 3. DFT band structures of monolayer $\mathrm{ZrI}_{2}$ (a), $\mathrm{MoTe}_{2}$ (b), and $\mathrm{WTe}_{2}$ (c) calculated without spin-orbit coupling. Bands close to the Fermi level have been plotted in blue to emphasize the different connectedness of the bands between $\mathrm{ZrI}_{2}$ and $\mathrm{MoTe}_{2}$ or $\mathrm{WTe}_{2}$.

studies in Fig. 4. To further clarify $D_{k}$, we consider only the crossings between the topmost, filled band and the bottommost, unfilled band, as highlighted by blue squares in Figs. 4(b)-4(e); i.e., we discard crossings that may occur between two filled bands, and also those between two unfilled bands [e.g., red circles in Fig. 4(e)].

To characterize the symmetry representation of the filled states, let us denote by $N_{+, k}\left(N_{-, k}\right)$ the number of filled, even (odd) Bloch states at $\boldsymbol{k}$ along either screw line, where the even (odd) representation is defined to have screw eigenvalue $+\exp \left(-i k_{x} / 2\right)\left[-\exp \left(-i k_{x} / 2\right)\right]$; $N_{+, k}+N_{-, k}=f$, and where $N_{+, k} \neq N_{-, k}$, we say that bands are inverted at $\boldsymbol{k}$, as exemplified by the interval $0 \leq k_{x}<k_{x}^{\prime}$ in Fig. 4(b). $N_{ \pm, k}$ relate to the total Dirac count through

$$
D_{k}=\frac{\left|N_{+, k}-N_{-, k}\right|}{2}+2 n_{k}, \quad n_{k} \in \mathbb{Z}^{\geq} .
$$

That is, $n_{k}$ belongs to the set of non-negative integers and is undeducible solely from $N_{ \pm, \boldsymbol{k}}$. To prove Eq. (1) for $\boldsymbol{k}$ along $Х \Gamma X$, recall that each connected pair of bands [where, again, each pair corresponds to two eigenvalue branches of $\bar{C}_{2 x}$ that unavoidably cross in the interpolation $\left.\left.k_{x} \rightarrow k_{x}+2 \pi\right)\right]$ is time-reversal degenerate at $X$, with each degenerate subspace comprising a single odd $\left(\bar{C}_{2 x}=+i\right)$ state and a single even state with $\bar{C}_{2 x}=-i$. Given an evenintegral filling $(f \in 2 \mathbb{Z})$, there are then an equal number $(f / 2)$ of filled even and filled odd states at $X$. Since each band is smoothly parametrized by its $\bar{C}_{2 x}$ eigenvalue, each filled state at $X$ must continuously interpolate, in the direction of decreasing $k_{x}$, to a state at $\boldsymbol{k}$; this interpolation occurs in the same branch of $\bar{C}_{2 x}$, but the final state at $k$ may 


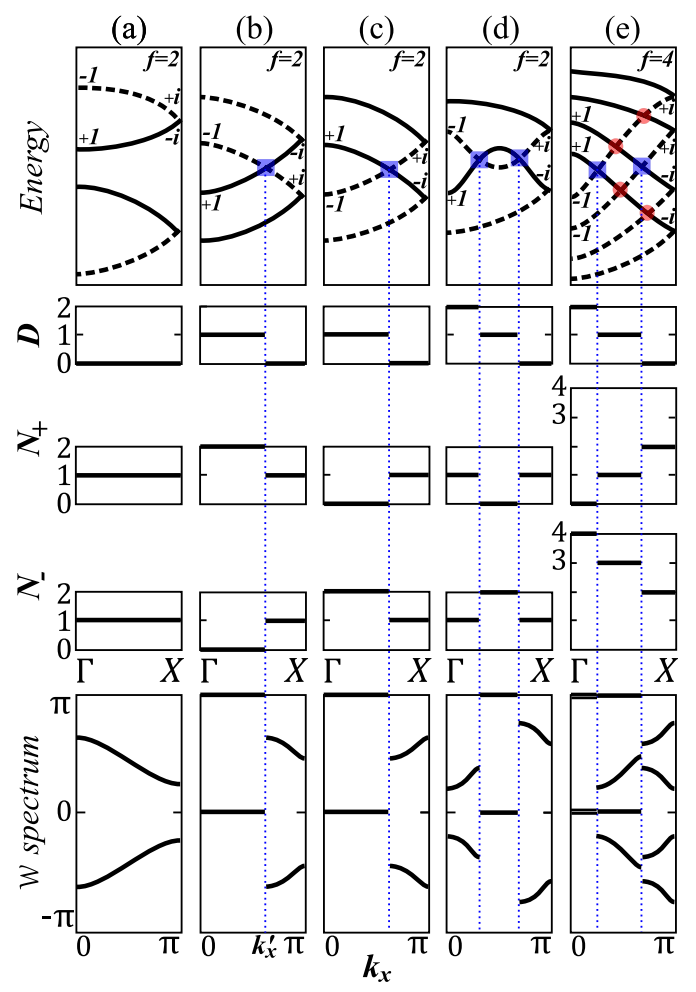

FIG. 4. Characterization of a generic insulator (a) and various topological metals (b)-(e). Top row: Band structures along the screw line $\Gamma X$. Solid (dashed) lines correspond to bands in the odd (even) screw representation, i.e., having screw eigenvalue $+\exp \left(-i k_{x} / 2\right)\left[-\exp \left(-i k_{x} / 2\right)\right]$. For each of (a)-(e), the filling $(f)$ is indicated in the top right-hand corner, and we assume that there are no Dirac crossings along the other screw line $Y M$. Blue circles denote crossings between a filled and an unfilled band, whereas red circles mark crossing between bands that are both filled (unfilled). Second, third, and fourth rows: Respectively, the numbers of Dirac crossings $\left(D_{k}\right)$, of even, filled states $\left(N_{+, k}\right)$, and of odd, filled states $\left(N_{-, k}\right)$, for $\boldsymbol{k}$ along $\Gamma X$. Bottom row: Eigenvalue phases of the $\mathcal{W}\left(k_{x}\right)$ spectrum.

or may not be filled. If $N_{+, k}=N_{-, k}=f / 2$, there are as many odd (even) states (at $\boldsymbol{k}$ ) as there are odd (even) states (at $X$ ) - then it is possible for the interpolation to occur entirely among the filled states, as exemplified by Fig. 4(a). However, if $N_{+, k}>N_{-, k}$, then a number $\left(N_{+, k}-N_{-, k}\right) / 2$ of filled states (at $X$ ) must interpolate to unfilled states (at $\boldsymbol{k}$ ), giving rise to $\left(N_{+, \Gamma}-N_{-, \Gamma}\right) / 2$ chiral modes in the even branch of $\bar{C}_{2 x}$, as illustrated for $N_{+, \Gamma}-N_{-, \Gamma}=1$ and 2 , respectively, in Figs. 4(c) and 4(e). By a similar demonstration, the same number of odd, antichiral modes must interpolate between filled states at $\boldsymbol{k}$ to unfilled states at $X$. This leads to minimally $\left(N_{+, k}-N_{-, k}\right) / 2$ screw-protected, Dirac crossings between chiral and antichiral modes, as highlighted by blue squares in Figs. 4(c) and 4(e). If instead $N_{+, k}<N_{-, k}$, an analogous demonstration leads to minimally $\left(N_{-, k}-N_{+, k}\right) / 2$ Dirac crossings [e.g., Fig. 4(b)]. We qualify $\left|N_{+, k}-N_{-, k}\right| / 2$ as the minimal Dirac count $\left(\bar{D}_{k}\right)$, because the total count $\left(D_{k}\right)$ can, in principle, be greater than $\bar{D}_{k}$ by any positive even number, due to band inversions away from $\boldsymbol{k}$; e.g., Fig. 4(d) illustrates two Dirac crossings along $\Gamma X$ (hence, $D_{\Gamma}=2$ ) despite $N_{+, \Gamma}=N_{-, \Gamma}$. This completes our proof of Eq. (1) for $\boldsymbol{k}$ along $Х \Gamma X$; the proof for $\boldsymbol{k}$ along $M Y M$ is obtained by cosmetically substituting $\Gamma \rightarrow Y$ and $X \rightarrow M$ in the above demonstration.

Since the above results depend essentially on the integerspin representation of time-reversal and screw symmetries, they (and the topological characterization described in the next section) would also apply to intrinsically spinless systems such as photonic crystals [49,50], though certain terms that naturally describe Fermi systems have to be reinterpreted. While "filling"is conventionally associated with Pauli exclusion, we may, at each wave vector, distinguish between filled and unfilled photonic bands separated by a frequency gap. There is, of course, no photonic metal in the sense of charge transport, though we may still discuss Dirac-type touchings between filled and unfilled bands.

\section{Topological characterization of band-inverted metals}

Each screw-protected Dirac touching is associated with a $\pi$ quantized Berry phase, which is acquired in traversing a screw-symmetric momentum loop around the Dirac node. More generally, we consider the parallel transport of filled Bloch waves around a momentum loop $l$, where at each $k \in l$ a spectral gap separates a set of lower-energy, filled states (numbering $f$ ) from a higher-energy, unfilled subspace. The $f$-by- $f$ matrix representing such parallel transport is known as the Wilson loop [51], and it may be expressed as the path-ordered exponential (denoted by exp) of the Berry-Wilczek-Zee connection [6,51] $\boldsymbol{A}(\boldsymbol{k})_{i j}=\left\langle u_{i, \boldsymbol{k}} \mid \nabla_{\boldsymbol{k}} u_{j, \boldsymbol{k}}\right\rangle$ :

$$
\mathcal{W}[l]=\exp \left[-\int_{l} d \boldsymbol{k} \cdot \boldsymbol{A}(\boldsymbol{k})\right],
$$

where $\left|u_{j, k}\right\rangle$ is an occupied eigenstate of the tight-binding Hamiltonian. The $U(f)$ Berry gauge field $(\boldsymbol{A})$ may be decomposed into trace-ful and trace-less components, where the trace-ful term $(\operatorname{Tr}[\boldsymbol{A}])$ generates the Abelian component of the parallel transport:

$$
e^{i \Phi_{\mathrm{U}(1)}[l]} \equiv \exp \left[-\int_{l} d \boldsymbol{k} \cdot \operatorname{Tr}[\boldsymbol{A}(\boldsymbol{k})]\right]=\operatorname{det}[\mathcal{W}[l]] .
$$

The $\mathrm{U}(1)$ Berry phase $\left(\Phi_{\mathrm{U}(1)}[l]\right)$ is quantized to $\pi$ if we choose $l$ to encircle the Dirac node screw symmetrically. By a "screw-symmetric circle,"we mean that $l$ [exemplified by $l_{1}$ and $l_{2}$ in Fig. 2(c)] is contractible, and is mapped to $-l$ by $\bar{C}_{2 x}$, where the sign of $l$ indicates its orientation; the 
mapping follows from $\bar{C}_{2 x}: y \rightarrow-y$ in real space, and, therefore, $k_{y} \rightarrow-k_{y}$ in momentum space. More generally, given any symmetry $(g)$ that maps $l \rightarrow-l$ and has a unitary representation $\left(\breve{g}^{-1}=\breve{g}^{\dagger}\right)$, then [15]

$$
\breve{g} \mathcal{W}[l] \breve{g}^{-1}=\mathcal{W}[-l]=\mathcal{W}[l]^{-1} \Rightarrow \operatorname{det}[\mathcal{W}[l]]= \pm 1 .
$$

The left-hand equation implies that the spectrum of $\mathcal{W}[l]$ is invariant under complex conjugation; the right-hand equation describes the quantization of the $U(1)$ Berry phase, which is robust against any deformations of the Hamiltonian that preserve both the symmetry $(g)$ and the spectral gap along $l$. In our context, $g=\bar{C}_{2 x}$, and $\exp \left(i \Phi_{\mathrm{U}(1)}[l]\right)=1(-1)$ if $l$ encircles an even (odd) number of Dirac fermions.

Our topological discussion has thus far focused on characterizing individual Dirac nodes, by an Abelian Berry-type invariant defined over contractible momentum loops; a global characterization of all Dirac nodes is possible with a non-Abelian Berry invariant $[15,18]$ defined over a noncontractible [2] momentum loop. By a nonAbelian Berry invariant, we mean that it requires knowledge of the individual eigenvalues of $\mathcal{W}[l]$, which encode the non-Abelian transport generated by the trace-less component of $\boldsymbol{A}$.

Henceforth, we consider only screw-symmetric loops $l\left(k_{x}\right)$ parallel to $\boldsymbol{e}_{y}$ and at fixed $k_{x}$, as illustrated in Fig. 2(c); we thus shorten $\mathcal{W}\left[l\left(k_{x}\right)\right]$ to $\mathcal{W}\left(k_{x}\right)$. Applying Eq. (4) for $l\left(k_{x}\right)$, the invariance of the $\mathcal{W}\left(k_{x}\right)$ spectrum under complex conjugation implies that a (possibly zero) subset of $\mathcal{W}\left(k_{x}\right)$ eigenvalues [numbering $W_{ \pm}\left(k_{x}\right)$ ] is, respectively, quantized to \pm 1 . In Appendix $A$, we relate these quantized $\mathcal{W}\left(k_{x}\right)$ eigenvalues to the total number $\left(D_{l\left(k_{x}\right)}\right)$ of Dirac crossings in the cylinder bounded by $l\left(k_{x}\right)$ and $M X M$ [red shaded region in Fig. 2(c)]:

$$
\begin{aligned}
D_{l\left(k_{x}\right)} & =\max \left\{W_{+}\left(k_{x}\right), W_{-}\left(k_{x}\right)\right\}+2 b, \\
b & \in \mathbb{Z}^{\geq} .
\end{aligned}
$$

As described in Sec. III B, such crossings can originate from independent band inversions on either of the two screw-invariant intervals to the right of $l\left(k_{x}\right)$ [marked as dashed lines in Fig. 2(c)]. The difference in Dirac counts between the two intervals also relates to the quantized $\mathcal{W}$ eigenvalues as

$$
D_{\left(k_{x}, 0\right)}-D_{\left(k_{x}, \pi\right)}-\min \left\{W_{+}\left(k_{x}\right), W_{-}\left(k_{x}\right)\right\} \in 2 \mathbb{Z},
$$

where $2 \mathbb{Z}$ denotes the set of even integers. Additional contributions to $D_{l\left(k_{x}\right)}$ may arise from screw-symmetric pairs of crossings away from screw lines, as may be stabilized by a spatial symmetry (e.g., inversion) other than screw.

We exemplify our result for crystals with fillings 2 and 4; their possible screw representations, $\mathcal{W}$ spectra and minimal Dirac counts are tabulated in Table I (in this section) and Table II (in Appendix A). These properties are then applied to topologically distinguish the different phases in Figs. 4(a)-4(e), which are all assumed to have no Dirac crossings along the unillustrated screw line $Y M$, and also no crossings away from the screw lines. We exemplify this analysis for Fig. 4(b), focusing on the interval $0 \leq k_{x}<k_{x}^{\prime}$. The filled bands are inverted in this segment of $\Gamma X$, with $N_{+,\left(k_{x}, 0\right)}=2, \quad N_{-,\left(k_{x}, 0\right)}=0$ by assumption of $Y M, N_{+,\left(k_{x}, \pi\right)}=N_{-,\left(k_{x}, \pi\right)}=1$. Thus, reading off the third-from-the-bottom row in Table I, we obtain $W_{ \pm}\left(k_{x}\right)=1 \quad$ [illustrated in the bottommost plot of Fig. 4(b)]. This further implies from Eq. (5) that the total Dirac count $D_{\left(k_{x}, 0\right)}+D_{\left(k_{x}, \pi\right)}=1+2 c$, with $c \in \mathbb{Z}^{\geq}$, and from Eq. (6) that both $D_{\left(k_{x}, 0\right)}$ and $D_{\left(k_{x}, \pi\right)}$ have the same parity; Fig. 4(b) shows, in fact, that $D_{\left(k_{x}, 0\right)}=1$ and by assumption $D_{\left(k_{x}, \pi\right)}=0$. Finally, we remark that the Wilson loop in Eq. (2) is only well defined and continuous on intervals that exclude the Dirac points; e.g., the discontinuity of $W_{ \pm}\left(k_{x}\right)$ at $k_{x}^{\prime}$ in Fig. 4(b) necessarily indicates a Dirac crossing. Our schematic example in Fig. 4(b) is further materialized by the spin-orbit-free $\mathrm{WTe}_{2}$ monolayer, as we demonstrate in Sec. IVA.

TABLE I. Topological characterization of a nonsymmorphic crystal with filling $f=2$ and either screw or glide symmetry. $\boldsymbol{k}_{1}$ and $\boldsymbol{k}_{2}$ are shorthand for the two screw-invariant (or glideinvariant) momenta at a particular $k_{x}:\left(k_{x}, 0\right)$ and $\left(k_{x}, \pi\right)$. Leftmost column: $N_{+, \boldsymbol{k}_{1}}$ is the number of filled states at $\boldsymbol{k}_{1}$ in the even screw (glide) branch, i.e., with screw (glide) eigenvalue $+\exp \left(-i k_{x} / 2\right) ; N_{-, k_{1}}=f-N_{+, k_{1}}$ is the number of odd, filled states with screw (glide) eigenvalue $-\exp \left(-i k_{x} / 2\right)$. Second column from the left: Number of even filled states at $\boldsymbol{k}_{2}$. Third column: $\mathcal{W}\left(k_{x}\right)$ spectrum, with $+(-)$ representing a $+1(-1)$ eigenvalue, and $\lambda_{i} \lambda_{i}^{*}$ a unimodular, complex-conjugate pair. Fourth column: The minimal number $\left(\equiv \bar{D}_{l\left(k_{x}\right)}\right)$ of Dirac crossings in a momentum cylinder bounded by $l\left(k_{x}\right)$ and $M X M$. Rightmost column: The parity of the difference in Dirac counts along either screw intervals. We remark that the $\mathcal{W}\left(k_{x}\right)$ spectrum depends only on relative changes in the symmetry representations between $\boldsymbol{k}_{1}$ and $\boldsymbol{k}_{2}$; i.e., it is invariant under (i) interchanging $N_{+, \boldsymbol{k}_{1}}$ with $N_{+, \boldsymbol{k}_{2}}$ and (ii) multiplication of all screw (glide) eigenvalues by a common factor -1 , i.e., $\left\{N_{+, k_{1}}, N_{+, k_{2}}\right\} \rightarrow$ $\left\{N_{+, \boldsymbol{k}_{1}}^{\prime}=f-N_{+, \boldsymbol{k}_{1}}, N_{+, \boldsymbol{k}_{2}}^{\prime}=f-N_{+, \boldsymbol{k}_{2}}\right\}$. For example, the $\mathcal{W}\left(k_{x}\right)$ spectrum and Dirac counts in the third-to-last row additionally describe a crystal with two odd states at $\boldsymbol{k}_{1}$ and an odd or even pair at $\boldsymbol{k}_{2}$, and also describe a crystal with two odd states at $\boldsymbol{k}_{2}$ and an odd or even pair at $\boldsymbol{k}_{1}$.

\begin{tabular}{lcccc}
\hline \hline$N_{+, \boldsymbol{k}_{1}}$ & $N_{-, \boldsymbol{k}_{2}}$ & $\mathcal{W}\left(k_{x}\right)$ & $\bar{D}_{l\left(k_{x}\right)}$ & $D_{\boldsymbol{k}_{1}}-D_{\boldsymbol{k}_{2}} \bmod 2$ \\
\hline 1 & 1 & {$\left[\lambda_{1} \lambda_{1}^{*}\right]$} & 0 & 0 \\
2 & 1 & {$[+-]$} & 1 & 1 \\
2 & 0 & {$[--]$} & 2 & 0 \\
2 & 2 & {$[++]$} & 2 & 0 \\
\hline \hline
\end{tabular}


Given the well-known U(1) ambiguity of the Wilson loop originating from the choice of real-spatial origin [15], all other higher-than-one-dimensional band topologies (with one exception [5]) are diagnosed by gauge-invariant differences of Wilson loops, between different 1D submanifolds in the same Brillouin zone (BZ) [15-19,52]. On the other hand, we propose that the invariants in Eqs. (5) and (6), as extracted from a single Wilson loop, are predictive of the Dirac metallicity in a $2 \mathrm{D}$ submanifold of the Brillouin zone-since the existence of Dirac points cannot depend on the choice of origin, our invariants are likewise independent, as we proceed to demonstrate. Since the Wilson loops we consider traverse a momentum path that is parallel to $\boldsymbol{e}_{y}$, their eigenspectrum depends on only the $y$ coordinate of the origin. Specifically, translating the spatial origin by $\delta \boldsymbol{r}$ induces a global phase shift of all $\mathcal{W}$ eigenvalues by $\boldsymbol{G} \cdot \delta \boldsymbol{r}$, with $\boldsymbol{G}$ the reciprocal period along $\boldsymbol{e}_{y}$ [15]. In a 2D Bravais lattice, there are always two inequivalent, real-spatial lines that are invariant under the screw rotation, as exemplified by horizontal dashed lines in Fig. 2(a) for the $\mathrm{WTe}_{2}$ monolayer; assuming no spatial symmetry other than screw, the spatial origin may lie at any point on either screw line. Since the two lines are separated by half a lattice period in $\boldsymbol{e}_{y}$, translating the origin between these lines induces a global phase shift of $\boldsymbol{G} \cdot \delta \boldsymbol{r}= \pm \pi$. Consequently, $W_{ \pm} \rightarrow W_{\mp}$, but their maximum and minimum values in Eqs. (5) and (6) are clearly invariant. We call any such quantity, that is both extractable from a single Wilson loop and insensitive to the spatial origin, a strong Wilson-loop invariant; another known example classifies a newly introduced nonsymmorphic topological insulator [5]; all other known single-Wilson-loop invariants $[2,15,19]$ are comparatively weak, and relate to spatially dependent physical predictions. A case in point is the geometric theory of polarization [53], which predicts different electronic charges at the edge of a crystal, depending on where the edge is terminated [54].

An experimental signature of the band-inverted topological metal is the presence of edge states that connect Dirac points in pairs, as we exemplify with $\mathrm{WTe}_{2}$ in Sec. IV B.

\section{Proximity of topological metals to the 2D $\mathbb{Z}_{2}$-topological insulator}

Topological metals are often linked to gapped, topological phases. There are various ways to arrive at such a gapped phase: (i) the mutual annihilation of band crossings with zero net topological charge, (ii) the breaking of a spatial symmetry, and (iii) the introduction of spin-orbit coupling in electronic metals [9], which may also be interpreted as a breaking of spin-SU(2) symmetry. To exemplify (i)-(iii) in this order, inversion-asymmetric Weyl metals intermediate between a trivial, gapped phase and a $\mathbb{Z}_{2}$-topological, gapped phase [55]; certain 3D Dirac semimetals [56] are gapped when their protective spatial symmetry is broken, leading to a novel, nonsymmorphic topological phase [57]; the slightest spin-orbit coupling gaps graphene [58], a symmorphic Dirac semimetal, to form a quantum spin Hall phase with $\mathbb{Z}_{2}$ topological order $[4,42,43]$ (in short, a $\mathbb{Z}_{2}$-topological insulator).

This section describes how, analogously to graphene, the slightest spin-orbit coupling gaps a nonsymmorphic Dirac metal to form a $\mathbb{Z}_{2}$-topological insulator. Not all Dirac metals necessarily lead to a $\mathbb{Z}_{2}$-topological phase when gapped-the criterion on the metal may be stated in three equivalent ways. If and only if the nonsymmorphic metal is characterized by (i) an odd number of Dirac fermions (per spin component) in half the Brillouin zone [red shaded region labeled by $\tau_{1 / 2}$ in Fig. 5(a)], or, equivalently, (ii) the Abelian component of the single-spin Wilson loop along $Y \Gamma Y$ [i.e., $\operatorname{det}[\mathcal{W}(0)]]$ equals -1 , or, equivalently, (iii) the product of the nonsymmorphic eigenvalues (whether glide or screw) of single-spin filled states over $\Gamma$ and $Y$ equals -1 , then any gap-inducing, spin-orbit coupling that preserves the nonsymmorphic symmetry (whether glide or screw) results in a $\mathbb{Z}_{2}$-topological phase.

More precisely, the above criterion guarantees the $\mathbb{Z}_{2}{ }^{-}$ topological phase for weak spin-orbit coupling; in principle, one cannot rule out that strong spin-orbit coupling might induce a transition to a trivial, gapped phase. That (i) and (ii) are equivalent follows from Eq. (5), where we deduce that odd $D_{l(0)}$ is equivalent to either odd $W_{+}(0)$ or odd $W_{-}(0)$. Because of the assumed even filling and the invariance of the $\mathcal{W}(0)$ spectrum under complex conjugation [cf. Eq. (4)], it is always the case that if either is odd, both $W_{+}(0)$ and $W_{-}(0)$ are odd, which leads to $\operatorname{det}[\mathcal{W}(0)]=-1$. That (i) and (iii) are equivalent follows from Eq. (1), which leads to

$$
D_{l(0)} \sim D_{\Gamma}+D_{Y} \sim \frac{\left|N_{+, \Gamma}-N_{-, \Gamma}\right|}{2}+\frac{\left|N_{+, Y}-N_{-, Y}\right|}{2},
$$

where $\sim$ denotes an equality modulo 2 . On further application of $f=N_{+, k}-N_{-, k} \in 2 \mathbb{Z}$, we deduce that odd $D_{l(0)}$ occurs if and only if $N_{-, \Gamma}+N_{-, Y}$ is also odd; finally, observe that the product of nonsymmorphic

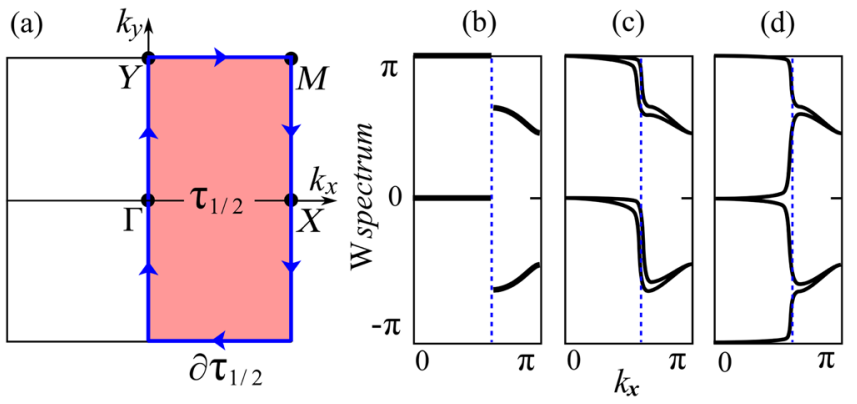

FIG. 5. (b)-(d) Pictorial argument for a quantum spin Hall phase, given a metal with a single Dirac fermion (per spin component) in $\tau_{1 / 2}$ [red shaded half-Brillouin zone in (a)]. 


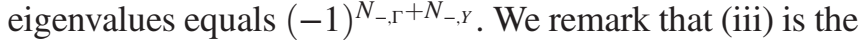
nonsymmorphic generalization of the Fu-Kane criterion [59] for a $\mathbb{Z}_{2}$-topological phase in centrosymmetric crystals.

To pictorially argue for our criterion, we return to our metallic case study in Fig. 4(b), which has only a single Dirac fermion along $\Gamma X$ and no other Dirac crossing in the cylinder bounded by $Y \Gamma Y$ and $M X M$. The filled states are characterized by $N_{-, \Gamma}=2, N_{+, \Gamma}=0$ and $N_{+, Y}=N_{-, Y}=1$, which implies that the product of their screw eigenvalues equals -1 . The third-from-the-bottom row of Table I informs us that the two $\mathcal{W}(0)$ eigenvalues (per spin component) are +1 and -1 , which implies that $\operatorname{det}[\mathcal{W}(0)]=-1$. For $0 \leq k_{x} \leq \pi$, the spectrum of $\mathcal{W}\left(k_{x}\right)$ is reproduced in Fig. 5(b), where each Wilson "band" is spin degenerate and discontinuous at the momentum position of the Dirac fermion (indicated by the blue dashed line). The introduction of spin-orbit coupling splits this spin degeneracy everywhere except for the Kramers degeneracies at the time-reversal-invariant $k_{x} \in\{0, \pi\}$. Additionally, the gapping of the Dirac fermion implies that a subspace of filled states is now smoothly defined over the entire Brillouin zone-this smoothens out the discontinuity in the $\mathcal{W}\left(k_{x}\right)$ spectrum. If we only assume that the spin-orbit coupling is time-reversal symmetric, there are two ways of smoothening: Fig. 5(c) [Fig. 5(d)] illustrates the Kramers partner-preserving (partner-switching [16,17,52]) doublets of the trivial gapped phase (the $\mathbb{Z}_{2}$-topological phase). If we further assume that the spin-orbit coupling respects the nonsymmorphic symmetry (whether glide or screw), then the $\mathcal{W}\left(k_{x}\right)$ spectrum is further constrained to be invariant under complex conjugation [cf. Eq. (4)], which uniquely selects the $\mathbb{Z}_{2}$-topological phase of Fig. 5(d). Beyond this pictorial argument, a technical proof of our criterion is provided in Appendix B.

Our criterion is more broadly predictive of $\mathbb{Z}_{2}$-topological phases which are metallic from the perspective of transport, so long as a finite energy difference exists between two sets of bands for all $\boldsymbol{k}$ - the time-reversal $\mathbb{Z}_{2}$ invariant [4] is then well defined for both sets of bands. This qualifier is relevant to spin-orbit-coupled $\mathrm{WTe}_{2}$ monolayers, which have $\mathbb{Z}_{2}$-topological order in conjunction with electron and hole pockets, as we demonstrate in Sec. IV D.

\section{E. Proximity of topological metals to the 3D $\mathbb{Z}_{2}$-topological insulator}

Our criterion for $\mathbb{Z}_{2}$-topological order in $2 \mathrm{D}$ is easily generalized to 3D nonsymmorphic insulators, which may be strongly or weakly topological. Supposing a 3D crystal is screw symmetric, we can always choose a Euclidean coordinate system such that the screw axis is parallel to $\boldsymbol{e}_{x}$. Then there are four time-reversal-invariant momenta in the plane $k_{x}=0$, which we label by $K_{j}$ with $j=1,2,3$, 4. Let us define the product of screw eigenvalues of single-spin filled states at $K_{j}$ as $\zeta_{j}$. Then, if the product $\prod_{j=1}^{4} \zeta_{j}=-1$, then spin-orbit coupling would induce a 3D strong topological insulator. If the product is +1 , spin-orbit coupling induces either a trivial gapped phase (if all of $\left\{\zeta_{j}\right\}$ have the same sign) or a weak topological insulator (if two of $\left\{\zeta_{j}\right\}$ have opposite sign from the other two). This criterion on screw eigenvalues may be equivalently formulated with the number of 3D Weyl fermions, or with the determinant of the Wilson loops. To derive this, we would apply our 2D criterion to time-reversal-symmetric planes in the 3D Brillouin torus.

For 3D crystals with a glide symmetry, we would choose coordinates such that the fractional translation is parallel to $\boldsymbol{e}_{x}$, and the reflection axis is orthogonal to $\boldsymbol{e}_{x}$. Then we need only consider the glide eigenvalues at the four time-reversal-invariant points in the plane $k_{x}=0$. The criterion for glide eigenvalues is identical to that for screw eigenvalues, as described in the previous paragraph. We remark that band inversion for glide-symmetric crystals results in line nodes within the glide-invariant planes, rather than the 3D Weyl fermions of screw-symmetric crystals.

\section{WTe 2 MONOLAYER}

In Sec. IV A, we present a minimal tight-binding model of a spin-orbit-free $\mathrm{WTe}_{2}$ monolayer as a model system, which confirms its topological metallicity in the sense of Sec. III B. This model also captures the tilting of the Dirac fermion, which we formalize in Sec. IV C by introducing the notion of a type-II Dirac fermion. Finally, in Sec. IV C, we apply our criterion in Sec. III D to predict that the spin-orbit-coupled $\mathrm{WTe}_{2}$ monolayer has $\mathbb{Z}_{2}$-topological order.

\section{A. Tight-binding model and topological characterization of the spin-orbit-free $\mathrm{WTe}_{2}$ monolayer}

We now present a minimal tight-binding model of the spin-orbit-free $\mathrm{WTe}_{2}$ monolayer, which reproduces the density functional theory (DFT) band structure near the Fermi level; compare Fig. 6(a) with Fig. 3(c). Our model includes the minimal number (four) of bands to describe a band inversion between two sets of twofold-connected bands; cf. Sec. III A. With modified tight-binding parameters, this model can be more generally be applied to $M X_{2}$ compounds with the same crystal structure.

By a Wannier interpolation [60] of these four DFT bands, we construct a basis of maximally localized Wannier functions, comprising two $d_{x^{2}-y^{2}}$-type orbitals that derive from the $\mathrm{W}$ atoms [indicated by $M$ in Fig. 2(a)] and two $p_{x}$-type orbitals derived from a subset of the Te atoms [X-2 in Fig. 2(a)]; there are no orbitals derived from the complementary Te sublattice $[X-1$ in Fig. 2(a)] in our lowenergy description. As indicated within the rectangular unit cell of Fig. 2(a), the centers of these Wannier functions divide into two sublattices, labeled by $A$ and $B$, which are 

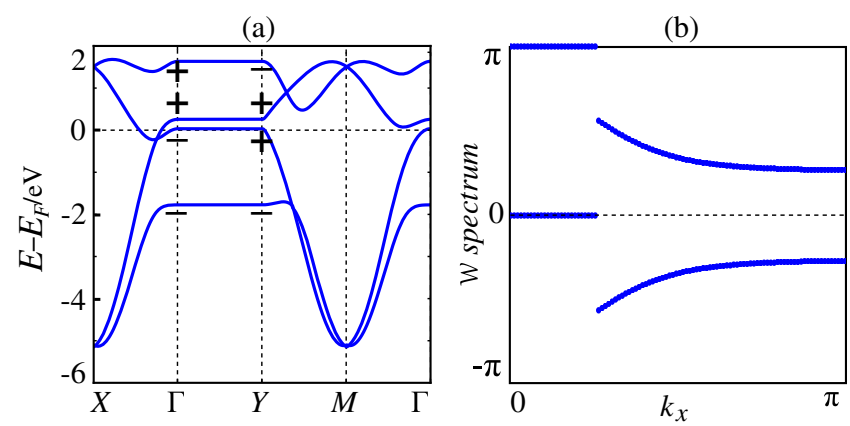

FIG. 6. (a) Band structure for the tight-binding model [Eq. (7)] of spin-orbit-free $\mathrm{WTe}_{2}$, with parameters listed in Appedix C. At $\Gamma$ and $Y$, + and - , respectively, label bands with screw eigenvalues +1 and -1 . (b) Phases of the Wilson-loop eigenvalues, as calculated for the same tight-binding model [Eq. (7)].

permuted by the screw transformation $\bar{C}_{2 x}$. Each $d_{x^{2}-y^{2}}$ type ( $p_{x}$-type) Wannier function is even (odd) under a mirror operation $x \mapsto-x$ centered at the $\mathrm{W}$ atom (Te atom).

In this reduced Hilbert space, our tight-binding Hamiltonian includes all symmetry-allowed, nearestneighbor hoppings, as well as two next-nearest-neighbor, intrasublattice hoppings along the chain:

$$
\begin{aligned}
H= & \sum_{\boldsymbol{R}}\left[\sum_{\ell} \mu_{\ell}\left(a_{\ell, \boldsymbol{R}}^{\dagger} a_{\ell, \boldsymbol{R}}+b_{\ell, \boldsymbol{R}}^{\dagger} b_{\ell, \boldsymbol{R}}\right)\right. \\
& +\sum_{\ell} t_{\ell}\left(a_{\ell, \boldsymbol{R}+\boldsymbol{e}_{x}}^{\dagger} a_{\ell, \boldsymbol{R}}+b_{\ell, \boldsymbol{R}+\boldsymbol{e}_{x}}^{\dagger} b_{\ell, \boldsymbol{R}}\right) \\
& +\sum_{n=0}^{1}(-1)^{n} t_{0}^{A B}\left(b_{p, \boldsymbol{R}+n e_{x}}^{\dagger} a_{d, \boldsymbol{R}}-b_{d, \boldsymbol{R}+n e_{x}}^{\dagger} a_{p, \boldsymbol{R}}\right) \\
& \left.+\sum_{\ell} \sum_{\boldsymbol{\delta}_{\ell}} t_{l}^{A B} a_{\ell, f+\boldsymbol{\delta}_{\ell}}^{\dagger} b_{\ell, \boldsymbol{R}}\right]+ \text { H.c. }
\end{aligned}
$$

Here, $\ell$ denotes the orbital type (either $p$ or $d$ ), $a_{\ell, \boldsymbol{R}}^{\dagger}\left(b_{\ell, \boldsymbol{R}}^{\dagger}\right)$ creates an $\ell$-type Wannier function centered at the position $r_{\ell, A}+\boldsymbol{R}\left(r_{\ell, B}+\boldsymbol{R}\right)$ as indicated in Fig. 2(a), and $\boldsymbol{\delta}_{\ell}$ are vectors given by $\boldsymbol{\delta}_{p}=\mathbf{0}, \boldsymbol{e}_{x}$ and $\boldsymbol{\delta}_{d}=\boldsymbol{e}_{x}+\boldsymbol{e}_{y}, \boldsymbol{e}_{y}$. Since our Hamiltonian is spin-SU(2) symmetric, we omit the spin label for each electron operator. The tight-binding parameters are listed in Appendix C, where we also express Eq. (7) in the momentum representation.

For

$$
t_{p}-t_{d}<\frac{\mu_{p}-\mu_{d}}{2}+\left(t_{p}^{A B}+t_{d}^{A B}\right)
$$

orthogonal screw representations at $\Gamma$ are inverted (i.e., $0=N_{+, \Gamma} \neq N_{-, \Gamma}=2$ ), as we illustrate in Fig. 6(a) for $\mathrm{WTe}_{2}$. This band inversion leads to a time-reversed pair of Dirac fermions along the screw line $X \Gamma X$, which are encoded as a discontinuity in the Wilson-loop spectra in
Fig. 6(b). These Dirac nodes necessarily belong to the screw line, but they can have any position along it; i.e., by perturbing the Hamiltonian, we might move the Dirac nodes along $X \Gamma X$.

\section{B. Edge-state signature of the topological metal}

A signature of the nontrivial topological phase is the presence of robust edge states, if the crystal is terminated in a manner that preserves the nonsymmorphic symmetry. These edge states connect the projected Dirac nodes of the bulk and can be detected by scanning tunneling microscopy experiments. In the case of $\mathrm{WTe}_{2}$, we terminate the crystal such that its edge is parallel to $\boldsymbol{e}_{x}$, and, therefore, there is a conserved wave vector $k_{x}$. The edge states must connect the two Dirac points along $\Gamma X$, and there are, in principle, two ways this might happen: the edge states lie in the momentum interval that contains either $k_{x}=0$ or $k_{x}=\pi$. Which of these two happens depends on the edge termination. Generally, we expect edges states at wave vectors where $\operatorname{det}\left[\mathcal{W}\left[l\left(k_{x}\right)\right]\right]=\pi$, if the edge is terminated with the same unit cell used in calculating $\mathcal{W}\left[l\left(k_{x}\right)\right]$. Indeed, a Wilsonloop eigenvalue of $\pi$ corresponds to Bloch-Wannier functions, which are extended as Bloch waves in $\boldsymbol{e}_{x}$, but maximally localized in $\boldsymbol{e}_{y}$ with a center located at the midpoint between two adjacent unit cells [5]; if the crystal is terminated at this midpoint, these BlochWannier functions hybridize to form edge states. To justify this argument, we plot in Fig. 7 the band structure of $\mathrm{WTe}_{2}$ in a ribbon geometry, with the edge unit cell chosen as in Fig. 2(a); this same unit cell is used in the Wilson-loop calculation of Fig. 6(b).

\section{Type-II Dirac fermions}

The Dirac crossing along the screw line $(\Gamma X)$ is described by an effective Hamiltonian:

$$
\mathcal{H}_{\mathrm{II}}(\boldsymbol{k})=u_{x} k_{x} \sigma_{0}+v_{x} k_{x} \sigma_{1}+v_{y} k_{y} \sigma_{2}
$$

to linear order in the momentum coordinates originating from the Dirac node, which we define as the point of degeneracy. $\sigma_{1,2}$ are Pauli matrices and $\sigma_{0}$ the identity matrix, in a pseudospin basis labeled by $\sigma_{1}| \pm\rangle= \pm| \pm\rangle$, with the orbital characters $| \pm\rangle=i \alpha_{ \pm}\left|p_{ \pm}\right\rangle+\beta_{ \pm}\left|d_{ \pm}\right\rangle$, where $\left|l_{ \pm}\right\rangle=(|l, A\rangle \pm|l, B\rangle) / \sqrt{2}$ are the bonding or antibonding combinations of $l$-type orbitals across the two sublattices. The greek symbols are real, normalized according to $\alpha_{ \pm}^{2}+\beta_{ \pm}^{2}=1$, and determined numerically by diagonalizing Eq. (7) at the Dirac node.

The group $(G)$ of this nodal wave vector [47] is generated by the screw $\bar{C}_{2 x}$ and the space-time inversion $T \mathcal{I}$ (i.e., the product of time reversal $T$ and spatial inversion $\mathcal{I}$ ), which, respectively, transform the Hamiltonian as 

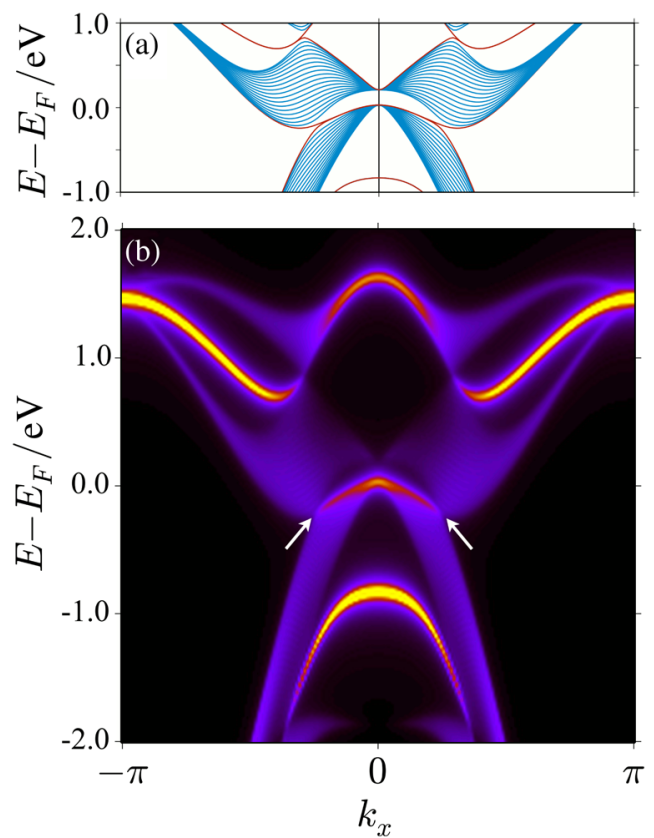

FIG. 7. (a) Band structure of a screw-symmetric ribbon of $\mathrm{WTe}_{2}$ calculated using the tight-binding model discussed in Sec. IV A. The ribbon is periodic in $\boldsymbol{e}_{x}$, and is 20 -unit-cell wide in $\boldsymbol{e}_{y}$. The edge states are highlighted in red. (b) Spectral function of the slab projected onto the first unit cell of the edge. Bright colors indicate a large contribution from the atoms located at the edge. The arrows indicate the Dirac points.

$$
\begin{aligned}
& \sigma_{1} \mathcal{H}_{\mathrm{II}}\left(k_{x}, k_{y}\right) \sigma_{1}=\mathcal{H}_{\mathrm{II}}\left(k_{x},-k_{y}\right), \\
& \sigma_{1} \mathcal{H}_{\mathrm{II}}^{*}\left(k_{x}, k_{y}\right) \sigma_{1}=\mathcal{H}_{\mathrm{II}}\left(k_{x}, k_{y}\right) .
\end{aligned}
$$

The lack of any discrete rotational symmetry in $G$ implies that the spectrum of $\mathcal{H}_{\mathrm{II}}$ is rotationally anisotropic, as evidenced by

$$
\epsilon_{ \pm}(\boldsymbol{k})=u_{x} k_{x} \pm \sqrt{v_{x}^{2} k_{x}^{2}+v_{y}^{2} k_{y}^{2}}
$$

The resulting anisotropy of the Fermi velocities may have important consequences in transport. This is in contrast to graphene, where each Dirac cone is fixed to a wave vector that is invariant under threefold rotation, such that the Dirac spectrum of graphene has an emergent, continuousrotational symmetry.

For $\mathrm{WTe}_{2}, G$ also lacks the reflection symmetry that maps $k_{x} \rightarrow-k_{x}$, as manifested by the allowed term $u_{x} k_{x} \sigma_{0}$ in the Hamiltonian. This term induces a tilting of the Dirac cone, which originates from the intrasublattice hoppings $t_{\ell}$ in Eq. (7). If the tilting is sufficiently pronounced, part of the upper Dirac cone dips below the nodal energy $\left[\epsilon_{ \pm}(\mathbf{0})=0\right]$, as illustrated in Fig. 1(a), resulting in a discontinuous change in the band contours at the nodal energy, i.e., a Lifshitz transition between a type-I $\left(\left|u_{x}\right|<\left|v_{x}\right|\right)$ spectrum with a closed Fermi circle surrounding $\boldsymbol{k}=0$ and a type-II $\left(\left|u_{x}\right|>\left|v_{x}\right|\right)$ spectrum with open Fermi line(s) to linear order in $\boldsymbol{k}$; the latter case applies to $\mathrm{WTe}_{2}$, as we illustrate in Figs. 1(a) and 1(b) [20,61]. All lattice-regularized Fermi surfaces are, of course, closed when higher-order momenta are accounted for-the regularized, type-II spectrum is described by two Fermi circles, with one being hole type and the other electron type. If the Fermi level lies at the type-II Dirac node [i.e., $\left.\varepsilon_{F}=\epsilon_{ \pm}(\mathbf{0})=0\right]$, the electron- and hole-type Fermi circles touch at the Dirac node. However, there is no symmetry constraint on the Fermi energy and so both circles are generically disconnected; for negative $\varepsilon_{F}$ (positive $\varepsilon_{F}$, as applies to $\mathrm{WTe}_{2}$ ), the hole-type (electron-type) Fermi circle is characterized by a $\mathrm{U}(1)$ Berry phase $\left[\Phi_{\mathrm{U}(1)}\right.$ in Eq. (3)] equal to $\pi$, while the electron-type (hole-type) Fermi circle has U(1) Berry phase equal to 0; this quantization is due to Eq. (3), where $l$ there corresponds to the Fermi circle described in this section.

We caution that the type-II Dirac fermions in monolayer $\mathrm{WTe}_{2}$ are not related to the predicted, type-II Weyl fermions in $3 \mathrm{D} \mathrm{WTe}_{2}$ [20], which have a different physical origin.

\section{Spin-orbit-coupled WTe $\mathrm{WT}_{2}$ monolayer}

The screw eigenvalues of the bands at $\Gamma$ and $Y$ are indicated in Fig. 6(a); their product over the filled bands equals -1 , which implies, through criterion (iii) in Sec. III D, that weakly-spin-orbit-coupled $\mathrm{WTe}_{2}$ has $\mathbb{Z}_{2^{-}}$ topological order. This result has consistently been derived [41] by exploiting the Fu-Kane criterion [59] for centrosymmetric crystals; we remark that our proposed criterion in Sec. III D more generally applies to nonsymmorphic metals without spatial-inversion symmetry.

\section{BILAYER}

We propose to interpret 3D WTe 2 as a periodic stacking of bilayers, for which the intrabilayer couplings dominate over the interbilayer couplings. We support our interpretation by finding that certain features of the 3D electronic structure, precisely the electron and hole pockets along the $\Gamma X$ line, are already present in the bilayer; we show in Sec. VA how these pockets (in both bilayer and 3D) ultimately originate from the tilted Dirac cones of the single monolayer. In Sec. V B, we further calculate the dichroism that originates from these pockets, so as to qualitatively explain the recently measured $\mathrm{CD}$ in $3 \mathrm{D} \mathrm{WTe}_{2}$ [38]. We limit ourselves to a qualitative description of the dichroism, and neglect quantitative corrections due to the weak spin-orbit coupling, which, as stated earlier, is an order of magnitude smaller than the typical tight-binding hopping parameter.

\section{A. Origin of electron and hole pockets in bilayer and 3D $\mathrm{WTe}_{2}$}

The electron and hole pockets of 3D $\mathrm{WTe}_{2}$ are a fundamental aspect of its electronic structure and are thought to be responsible for the large, nonsaturating 


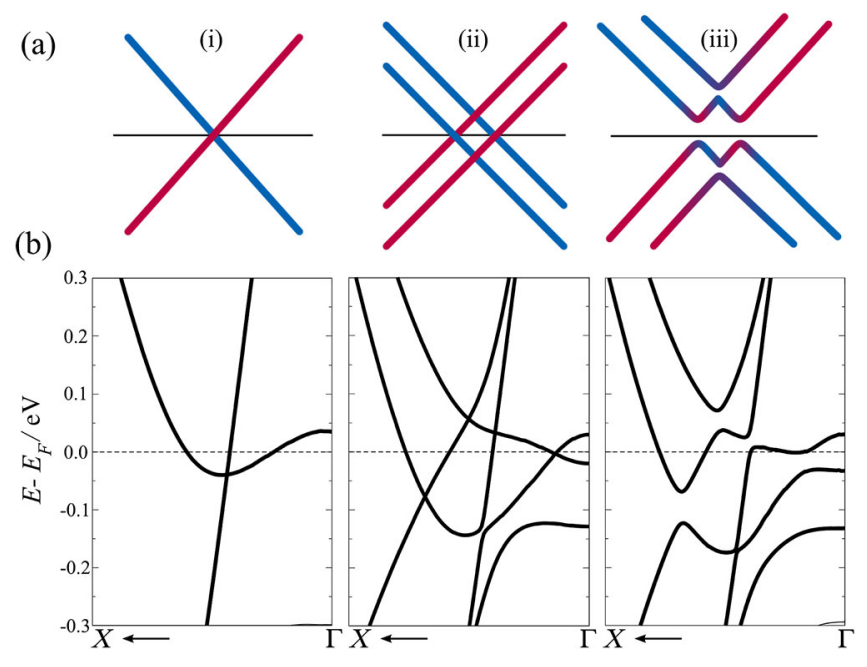

FIG. 8. (a) Hypothetical coupling of two band-inverted monolayers with type-I Dirac fermions: (a)(i) depicts a doubly degenerate Dirac crossing with vanishing coupling, (a)(ii) a metallic state with two distinct Dirac cones if the nonvanishing coupling preserves the screw symmetry, (a)(iii) a trivial insulator in the case of nonvanishing screw-asymmetric coupling. (b) DFT band structures of bilayer $\mathrm{WTe}_{2}$ for different intermonolayer couplings: (b)(i) vanishing, (b)(ii) screw-symmetry preserving, and (b)(iii) screw-symmetry breaking.

magnetoresistance [36]. Here, we propose that these pockets originate from the type-II Dirac fermions of the spin-orbit-free monolayer.

Our argument is illustrated in Fig. 8. To begin, in the limit of vanishing coupling between the monolayers, the bilayer band structure is doubly degenerate at all wave vectors. This follows because one monolayer is related to the other by a global continuous translation in real space, so that the energy-momentum dispersion curves of each monolayer are identical. We focus on a low-energy theory near the Dirac node, which is doubly degenerate in the aforementioned limit.

We now consider two different stackings for a bilayer of $\mathrm{WTe}_{2}$ : in the first, the two monolayers have no relative displacement in the $\boldsymbol{e}_{x}-\boldsymbol{e}_{y}$ plane but are displaced along $\boldsymbol{e}_{z}$ and, therefore, the bilayer retains the screw symmetry of the monolayer; we warn that this hypothetical stacking is unlikely to occur in nature. The intermonolayer coupling energetically splits the two Dirac cones, which correspond to bonding and antibonding combinations of the two monolayer wave functions, as illustrated in Fig. 8(b)(ii); the degeneracy of the Dirac crossing is retained since screw symmetry is preserved. The lowenergy theory of each Dirac crossing is described by the Hamiltonian in Eq. (9), albeit with slightly different parameters and basis wave functions. Independent of the particular basis, we note that Eq. (9) describes a pseudospin that is coupled to a pseudomagnetic field $(\boldsymbol{B})$ with components $B_{x}(\boldsymbol{k})=v_{x} k_{x}, B_{y}(\boldsymbol{k})=v_{y} k_{y}, B_{z}(\boldsymbol{k})=0$. The vanishing of $B_{z}$ is a consequence of the spatial symmetries which constrain the Hamiltonian as in Eq. (10)—-the Hamiltonian eigenfunctions therefore correspond to a pseudospin that is confined to a pseudo $x-y$ plane, and, moreover, winds as we encircle the Dirac node.

The second stacking corresponds to the experimental lattice parameters for $3 \mathrm{D} \mathrm{WTe}_{2}$, and breaks the screw symmetry, as we illustrate in Fig. 2(b). The asymmetric band structure differs from the symmetric stacking in that small gaps open at the Dirac nodes; compare Figs. 8(b)(ii) and (b)(iii). Otherwise, both band structures are very similar away from the Dirac nodes, and, in particular, both possess electron and hole Fermi pockets in the vicinity of the Dirac nodes. Each screw-asymmetric Dirac fermion is described by a small mass term proportional to $\sigma_{3}$; i.e., $B_{z}$ is a nonvanishingly small constant. Therefore, around either Fermi circle, the pseudospin rotates in the $x-y$ plane with a small out-of-plane component. In particular, the sense of rotation for the electronlike Fermi circle is opposite to that of the holelike circle, with consequences for the dichroism that we elaborate in Sec. V B.

We argue that these Fermi pockets are not generic and originate from the tilting of the Dirac cone. For the sake of this argument, we schematically illustrate in Fig. 8(a) a hypothetical bilayer that comprises two band-inverted monolayers. In this hypothetical scenario, the Dirac cone of the monolayer is type I and the Fermi surfaces are pointlike; therefore, screw-symmetry-breaking intermonolayer couplings generically produce a fully gapped, trivial insulator, as we argue pictorially through Fig. 8(a).

\section{B. Dichroism}

Circular dichroism has recently been observed in the ARPES of 3D $\mathrm{WTe}_{2}$ [38]; i.e., the photoemission is dependent on the helical polarization of light. CD depends on the wave function of the initial and the final electronic state upon photon absorption and is therefore a sensitive probe of the electronic structure of a material [64]. A simple and widely applied model of photoemission breaks down the process into three steps: (i) photoexcitation of an electron in the solid, (ii) propagation of the photoelectron to the surface, and (iii) escape of the photoelectron into the vacuum [65]. CD is encoded in the first step, where the photon causes a transition between two Bloch states in the solid. The intensity of the photoemitted electrons with wave vector $\boldsymbol{k}$ parallel to the surface and kinetic energy $E_{\text {kin }}$ due to photons of energy $\hbar \omega$ and polarization vector $\lambda$ is given by

$$
\begin{aligned}
I_{\lambda}\left(\boldsymbol{k}, E_{\mathrm{kin}}, \hbar \omega\right) \propto & \sum_{i, f}\left|P_{\lambda}^{i f}(\boldsymbol{k})\right|^{2} \delta\left(E_{f}-E_{i}-\hbar \omega\right) \\
& \times \delta\left(E_{\mathrm{kin}}-\left[E_{f}-\phi\right]\right) .
\end{aligned}
$$

Here, $P_{\lambda}^{i f}(\boldsymbol{k})=\langle f, \boldsymbol{k}|\boldsymbol{\lambda} \cdot \boldsymbol{p}| i, \boldsymbol{k}\rangle$ is the matrix element of the momentum operator $\boldsymbol{p}$ between initial $(i)$ and final $(f)$ 

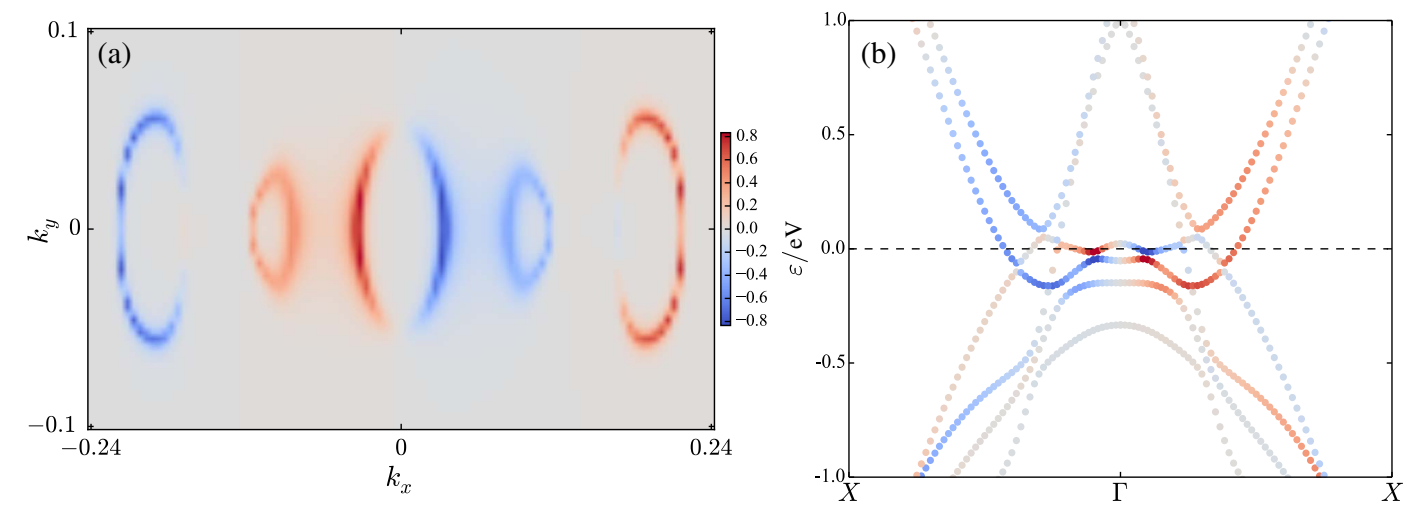

FIG. 9. (a) Fermi-level dichroic signal $\left[D_{s}\left(\boldsymbol{k}, \varepsilon_{F}\right)\right.$ defined in the main text] for a $\mathrm{WTe}_{2}$ bilayer. The Fermi surface has been broadened with a Lorentzian with a full width at half maximum of $10 \mathrm{meV}$. The Fermi pocket around $\Gamma[38,62,63]$ does not encircle a massive Dirac fermion. (b) $D_{s}(\boldsymbol{k}, E)$ for the band structure along $X \Gamma X$ for energies near the Fermi level.

Bloch states of energy $E_{i}$ and $E_{f}$, respectively, and $\phi$ is the work function [65].

In the simplest case of normal incidence along $\boldsymbol{e}_{z}$, the relevant matrix elements are $P_{ \pm}^{i f}(\boldsymbol{k})=\left\langle f, \boldsymbol{k}\left|p_{x} \pm i p_{y}\right| i, \boldsymbol{k}\right\rangle$, with the signs corresponding to the two different circular polarizations of the light. In this work, we ignore finalstate effects and focus on the dichroism that originates from initial states at the Fermi level; i.e., we study the Fermi-level dichroic signal defined by $D_{s}\left(\boldsymbol{k}, \varepsilon_{F}\right):=$ $I_{+}\left(\boldsymbol{k}, \varepsilon_{F}\right)-I_{-}\left(\boldsymbol{k}, \varepsilon_{F}\right)$. To disentangle initial- and final-state information from the experimental CD spectra, one typically needs to analyze the dependence of the spectra on the photon incidence angle and on the light energy [66-68]. In monolayer graphene, unambiguous information on the initial states can be extracted from the spectra because in this system the electronic wave function near the Fermi level corresponds to a pseudospin that winds around each Dirac point, resulting in dichroism for a narrow range of photon energies $[39,40]$.

We propose that the dichroism in bilayer $\mathrm{WTe}_{2}$ similarly originates from tilted Dirac fermions with small masses. As explained in Sec. VA, the pseudospin rotates around the electronlike Fermi circle and in the opposite sense around the holelike Fermi circle; thus, we expect that $D_{s}\left(\boldsymbol{k}, \epsilon_{F}\right)$ should carry opposite sign along the electronlike and the holelike Fermi circles [39]. To support this hypothesis, we calculate $D_{s}\left(\boldsymbol{k}, \varepsilon_{F}\right)$ using the DFT-derived initial-state wave functions, as further elaborated in Appendix D. As shown in Fig. 9(a), $D_{s}\left(\boldsymbol{k}, \varepsilon_{F}\right)$ is constrained by timereversal and $\bar{M}_{x}$ symmetries as $D_{s}\left(\boldsymbol{k}, \varepsilon_{F}\right)=-D_{s}\left(-\boldsymbol{k}, \varepsilon_{F}\right)$ and $D_{s}\left(k_{x}, k_{y}, \varepsilon_{F}\right)=-D_{s}\left(-k_{x}, k_{y}, \varepsilon_{F}\right)$, respectively. When the dichroic signal is strong, $D_{s}\left(\boldsymbol{k}, \varepsilon_{F}\right)$ shows the expected sign change between electron and hole Fermi circles; we note that the signal vanishes in a small momentum segment along the electron Fermi circle. Figure 9(b) further illustrates the nonuniform variation of the $D_{s}(\boldsymbol{k}, E)$ over a small range of energies.
We suggest also that the observed dichroism in $3 \mathrm{D} \mathrm{WTe}_{2}$ originates from the tilted, massive Dirac fermions. Quantitative comparison with experiment would need to account for final-state effects, spin-orbit coupling, as well as the 3D coupling between bilayers. We defer this to a future investigation. In closing this section, we note that ARPES and quantum oscillation experiments suggest the presence of a zone center Fermi pocket in 3D WTe 2 , which is absent in our DFT calculation, where it is pushed below the Fermi level. These fine effects are, however, very sensitive to small changes in the atomic parameters and functional approximations $[38,62,63]$.

\section{DISCUSSION}

The recent discovery of 3D Dirac and Weyl semimetals [69-80] has moved topological metals to the forefront of theoretical and experimental studies [81-89]. While Weyl nodes in 3D quasimomentum space require no symmetry beyond that of discrete translations, 2D Dirac nodes require an additional symmorphic space-time symmetry, which in graphene is a simultaneous inversion of space and time. The robustness of Weyl and Dirac points originates from quantized topological invariants that relate to the Abelian Berry gauge field of Bloch bands.

Among the predicted Weyl semimetals, 3D $M \mathrm{Te}_{2}$ $(M=\mathrm{W}, \mathrm{Mo})$ reveal a particularly rich range of phenomena: (i) they become superconducting under pressure [46,90-93], (ii) $\mathrm{WTe}_{2}$ demonstrates a giant, nonsaturating transverse magnetoresistance, and also (iii) circular dichroism in its photoemission [38]. In this work, we demonstrate that some of these exotic 3D properties may be extrapolated from topologically characterizing a single monolayer without SOC; this simplification is possible because 3D WTe 2 has weak SOC, and is, moreover, composed of weakly coupled monolayers.

We find that the spin-orbit-free $M \mathrm{Te}_{2}$ monolayer belongs to a new class of band-inverted metals, which are diagnosed 
by a topological invariant associated to a non-Abelian Berry gauge field, which contrasts with previous Abelian Berry-type characterizations of topological metals. The Dirac crossings of our metals rely on a nonsymmorphic symmetry; they differ from previously proposed, nonsymmorphic metals $[9,10]$ whose metallicity is guaranteed solely by the electron filling. The Dirac cones of $M \mathrm{Te}_{2}$ tilt over and are classified as type II. This has important implications for the band structure of 3D $M \mathrm{Te}_{2}$ : nearly compensated electron and hole pockets emerge, which encircle Dirac fermions with small masses; these pockets are characterized by a rotating pseudospin with consequences for circular dichroism.

Recent progress in the fabrication of few-layer $\mathrm{WTe}_{2}$ has enabled magnetoresistance measurements that support our theory of electron-hole compensation [94,95]. Our predicted dichroism has not been verified, but should now be possible to observe.

We further relate our findings to the giant, nonsaturating magnetoresistance observed in $3 \mathrm{D} M \mathrm{Te}_{2}$. The magnitude of the magnetoresistance in a two-band model with perfectly compensated electron and hole carriers is given by $[\rho(H)-\rho(0)] / \rho(0)=\mu_{e} \mu_{h} B^{2}$, where $\mu_{e}, \mu_{h}$ are the electron and hole mobilities [36,96]. The geometric mean of the mobilities has been extracted as $\sqrt{\mu_{e} \mu_{h}}=167000 \mathrm{~cm}^{2} / \mathrm{Vs}$ in $3 \mathrm{D} \mathrm{WTe}_{2}$ [37], as a result of the large magnetoresistance in this model. Additionally, the measured residual resistivity (RR) of $10^{-7} \Omega \mathrm{cm}$ is extremely low compared to other binary compounds. Applying a magnetic field of $1 \mathrm{~T}$ to $\mathrm{WTe}_{2}$ increases the low-temperature RR to the order of $10^{-4} \Omega \mathrm{cm}$ [97,98]. Extremely high mobilities are common to Dirac semimetals such as graphene, where the winding pseudospin suppresses backscattering from impurities and defects. We suggest that a similar mechanism suppresses backscattering in $3 \mathrm{D} \mathrm{WTe} 2$, which hosts massive, tilted Dirac fermions. Long relaxation times in transport, in combination with the low impurity concentration in measured crystals, may account for the large mobilities. The tilting of the Dirac cone hints at the strong anisotropy in the measured MR. However, a complete explanation of the large MR should account for the coupling of the magnetic field to the electronic structure, which is known to be nontrivial for other highMR materials, such as $\mathrm{Cd}_{3} \mathrm{As}_{2}$ [99], The nature of point defects in $\mathrm{WTe}_{2}$ and their effect on the electronic structure may be probed by quasiparticle interference and studied by computational methods.

We remark that electron-electron interactions modify the electron velocities such that they tend toward rotational isotropy at low energies. If screening is sufficiently weak, interactions may induce a type-II to type-I Lifshitz transition [32]. The strength of screening depends on the density of states at the Fermi level, which for spin-orbitfree $\mathrm{WTe}_{2}$ is not negligible.
We close this paper by remarking on the role of spatial symmetries in band topology. To date, all the proposed topological insulators that have been confirmed experimentally possess symmorphic spatial symmetry [100-104]. However, a material class has recently been proposed, whose band topology relies essentially on nonsymmorphic symmetries [48]. Moreover, an analogous band topology can also be realized in photonic crystals [105]. Nonsymmorphic spatial symmetries have also served to classify band metals $[9,10,106]$ and their Fermiliquid analogs [107], and have been used to identify topologically ordered insulators with fractionalized excitations [108-110]. We expect that our theory of bandinverted, nonsymmorphic metals, introduced here, may be generalized to include spin-orbit coupling, as we exemplify in Fig. 1(d).

\section{ACKNOWLEDGMENTS}

The authors would like to thank M. Ali, Q. Gibson, F. Tafti, W. Hu, L. Lin, T. Berkelbach, R. Cava, and D. Vanderbilt for helpful discussions, as well as M. Gibertini for critical help with WANNIER90. A. A. was supported by the Yale Postdoctoral Prize Fellowship, and in earlier stages by NSF CAREER DMR-095242, ONR-N00014-11-10635, MURI-130-6082, NSF-MRSEC DMR-0819860, Packard Foundation, Keck grant, "ONR Majorana Fermions" 25812-G0001-10006242-101, and Schmidt fund 23800-E2359-FB62. L. M. and R. C. were supported by the Department of Energy Grant No. DE-FG0205ER46201. The computations used the resources of the Terascale Infrastructure for Groundbreaking Research in Science and Engineering (TIGRESS) High Performance Computing Center and Visualization Laboratory at Princeton University.

L. M. and A. A. contributed equally as co-first authors in this work.

\section{APPENDIX A: TOPOLOGICALLY CLASSIFYING METALS THROUGH THE WILSON LOOP}

In this appendix, we relate the quantized $\mathcal{W}\left(k_{x}\right)$ eigenvalues [i.e., the \pm 1 eigenvalues that number $W_{ \pm}\left(k_{x}\right)$ ] to the minimal Dirac count $\left(\bar{D}_{k}\right)$, which counts the minimal number of Dirac crossings on either screw-invariant interval to the right of $l\left(k_{x}\right)$ [dashed lines in Fig. 2(c)]. Precisely, since there are two independent, minimal Dirac counts, corresponding to either the $k_{y}=0$ or $k_{y}=\pi$ screw lines, we would relate $W_{ \pm}\left(k_{x}\right)$ to their sum and absolute difference:

$$
\begin{aligned}
\bar{D}_{\left(k_{x}, 0\right)}+\bar{D}_{\left(k_{x}, \pi\right)} & =\max \left\{W_{+}\left(k_{x}\right), W_{-}\left(k_{x}\right)\right\} \\
\left|\bar{D}_{\left(k_{x}, 0\right)}-\bar{D}_{\left(k_{x}, \pi\right)}\right| & =\min \left\{W_{+}\left(k_{x}\right), W_{-}\left(k_{x}\right)\right\} .
\end{aligned}
$$

This derivation is intermediated by the relation (derived in Sec. III B) between the minimal Dirac count and the 
symmetry representations of filled states:

$$
\bar{D}_{k}=\frac{\left|N_{+, k}-N_{-, k}\right|}{2} .
$$

From Eqs. (1) and (A2), we further deduce Eq. (6) and, for $a \in \mathbb{Z}^{\geq}$,

$D_{\left(k_{x}, 0\right)}+D_{\left(k_{x}, \pi\right)}=\max \left\{W_{+}\left(k_{x}\right), W_{-}\left(k_{x}\right)\right\}+2 a$.

If a spatial symmetry other than screw symmetry (e.g., inversion) stabilizes Dirac crossings away from the screw lines, such crossings always exist in screw-symmetric pairs. Consequently, the total number $\left(D_{l\left(k_{x}\right)}\right)$ of Dirac crossings in the cylinder bounded by $l\left(k_{x}\right)$ and $l(\pi)$ [red shaded region in Fig. 2(c)] satisfies Eq. (5).

The remainder of this appendix aims to prove Eq. (A1). Let us denote by $g_{\delta}$ a spatial transformation, which transforms real-space coordinates as $\boldsymbol{r} \rightarrow D_{g} \boldsymbol{r}+\boldsymbol{\delta}$, where $D_{g}$ is the orthogonal matrix representation of the point-group transformation $g$ in $\mathbb{R}^{d}$. The nonsymmorphic space groups of interest to us contain symmetry elements for which $\delta$ is a rational fraction [7] of the lattice period. Each spatial symmetry constrains the Bloch Hamiltonian as [5]

$$
\hat{g}_{\boldsymbol{\delta}}(\boldsymbol{k}) H(\boldsymbol{k}) \hat{g}_{\boldsymbol{\delta}}(\boldsymbol{k})^{-1}=H\left(D_{g} \boldsymbol{k}\right),
$$

where the operator $\hat{g}_{\boldsymbol{\delta}}(\boldsymbol{k})$ may be decomposed into a phase factor and a unitary matrix as

$$
\hat{g}_{\boldsymbol{\delta}}(\boldsymbol{k}) \equiv e^{-i\left(D_{g} k\right) \cdot \boldsymbol{\delta}} U_{g \boldsymbol{\delta}}
$$

The set of all operators $\left\{\hat{g}_{\delta}(\boldsymbol{k})\right\}$ forms a representation of the space-group algebra [7] in a basis of Bloch-wavetransformed Löwdin orbitals [111]; in short, we call this the Löwdin representation. The nontrivial phase factor $\exp \left(-i D_{g} \boldsymbol{k} \cdot \boldsymbol{\delta}\right)$ in $\hat{g}_{\boldsymbol{\delta}}(\boldsymbol{k})$ encodes the effect of the fractional translation; i.e., the momentum-independent matrices $\left\{U_{g \delta}\right\}$ form by themselves a representation of a point group. A case in point is the screw rotation $\bar{C}_{2 x}$, which is a twofold rotation $\left(g=C_{2 x}\right)$ about $\boldsymbol{e}_{x}$, followed by a fractional lattice translation $t\left(\boldsymbol{\delta}=\boldsymbol{e}_{x} / 2\right)$ parallel to the rotational axis; $\bar{C}_{2 x}^{2}=t\left(\boldsymbol{e}_{x}\right)$, an integral lattice translation. $\bar{C}_{2 x}$ is a 3D symmetry of the $\mathrm{WTe}_{2}$ monolayers, which extend macroscopically in $\boldsymbol{e}_{x}$ and $\boldsymbol{e}_{y}$, and have finite, atomic-scale thickness in $\boldsymbol{e}_{z}$. In the 2D Brillouin zone parametrized by $\boldsymbol{k}=\left(k_{x}, k_{y}\right)^{t}$, the screw rotation maps $\boldsymbol{k} \rightarrow$ $\left(k_{x},-k_{y}\right)^{t} \equiv D_{2 x}\left(k_{x}, k_{y}\right)^{t}$. Identifying $D_{g}$ in Eq. (A5) with $D_{2 x}$, the Löwdin representation of $\bar{C}_{2 x}$ is

$$
\hat{\bar{C}}_{2 x}(\boldsymbol{k})=e^{-i k_{x} / 2} U_{2 x, \boldsymbol{e}_{x} / 2},
$$

which satisfies the nonsymmorphic algebra $\left[\bar{C}_{2 x}^{2}=t\left(\boldsymbol{e}_{x}\right)\right]$ of a screw rotation:

$$
\hat{\bar{C}}_{2 x}\left(D_{2 x} \boldsymbol{k}\right) \hat{\bar{C}}_{2 x}(\boldsymbol{k})=e^{-i k_{x}} .
$$

The momentum-independent unitary matrix $U_{2 x, \boldsymbol{e}_{x} / 2}$ forms a representation of a screwless twofold rotation; i.e., $U_{2 x, \boldsymbol{e}_{x} / 2}^{2}=I$. Substituting Eq. (A6) into Eq. (A4) yields the condition

$$
U_{2 x, \boldsymbol{e}_{x} / 2} H\left(k_{x}, k_{y}\right) U_{2 x, \boldsymbol{e}_{x} / 2}^{-1}=H\left(k_{x},-k_{y}\right),
$$

which is, for fixed $k_{x}$, mathematically equivalent to the Hamiltonian of a 1D crystal with spatial-inversion symmetry represented by $U_{2 x, \boldsymbol{e}_{x} / 2}$. This identification allows us to apply a known mapping between the spatial-inversion eigenvalues (of the occupied bands) and the Wilson-loop eigenvalues (for any constant- $k_{x}$ momentum loop, e.g., $Y \Gamma Y)$. This mapping may be found in Sec. II B of Ref. [15], where the inversion eigenvalues, \pm 1 , of filled states at the inversion-invariant momenta are now identified with the branches of screw eigenvalues $\left[ \pm \exp \left(-i k_{x} / 2\right)\right]$ of filled states at $\left(k_{x}, 0\right)$ and $\left(k_{x}, \pi\right)$. For the reader's convenience, we reproduce the mapping below.

Definition.-The filled states at each $\boldsymbol{k} \in\left\{\left(k_{x}, 0\right)\right.$, $\left.\left(k_{x}, \pi\right)\right\}$ divide into two sets (labeled by $\xi \in \pm 1$ ) according to the branch of their screw eigenvalues: $\xi \exp \left(-i k_{x} / 2\right)$. In short, we refer to $\xi$ as the screw branch, and further define the size of each set as $N_{\xi, k}$. Given the list $\left\{N_{+,\left(k_{x}, 0\right)}, N_{-,\left(k_{x}, 0\right)}, N_{+,\left(k_{x}, \pi\right)}, N_{-,\left(k_{x}, \pi\right)}\right\}$, we identify the smallest of these four integers and label it as $N_{\bar{\xi}, \bar{k}}$, where $\bar{\xi} \in \pm 1$ denotes the screw branch of this smallest set of states, and $\overline{\boldsymbol{k}} \in\left\{\left(k_{x}, 0\right),\left(k_{x}, \pi\right)\right\}$ its quasimomentum. We denote by $\overline{\boldsymbol{k}}_{c}$ the complementary quasimomentum. To recapitulate, we have mapped

$$
\begin{aligned}
& \left\{N_{+,\left(k_{x}, 0\right)}, N_{-,\left(k_{x}, 0\right)}, N_{+,\left(k_{x}, \pi\right)}, N_{-,\left(k_{x}, \pi\right)}\right\} \\
& \quad \rightarrow\left\{N_{\bar{\xi}, \bar{k}}, N_{-\bar{\xi}, \bar{k}}, N_{+, \overline{\boldsymbol{k}}_{c}}, N_{-, \overline{\boldsymbol{k}}_{c}}\right\} .
\end{aligned}
$$

The smallest set might be empty; in cases where the smallest set is not unique, any choice between "equally smallest" sets is valid. Let us exemplify the identification of the smallest set. (a) Consider a $f=2$ crystal with screw branches $(++)$ at $\left(k_{x}, 0\right)$ and $(+-)$ at $\left(k_{x}, \pi\right)$. The smallest set is then the empty set at $\left(k_{x}, 0\right)$ with screw branch -1 ; i.e., $N_{\bar{\xi}, \bar{k}}=0$, with $\bar{\xi}=-1$ and $\overline{\boldsymbol{k}}=\left(k_{x}, 0\right)$. (b) Suppose $f=4$ with screw branches $(++--)$ at $\left(k_{x}, 0\right)$ and $(+++-)$ at $\left(k_{x}, \pi\right)$, then $\overline{\boldsymbol{k}}=\left(k_{x}, \pi\right)$ and $\bar{\xi}=-1$. (c) For $f=2$ with screw branches $(+-)$ at $\left(k_{x}, 0\right)$ and $(+-)$ at $\left(k_{x}, \pi\right)$, we may arbitrarily pick any of the four possibilities as the smallest set.

While the identification of $N_{\bar{\xi}, \bar{k}}$ is simple by inspection, it is eventually worthwhile to express this quantity as 


$$
\begin{aligned}
N_{\bar{\xi}, \bar{k}}= & \frac{f-\left|N_{+, \overline{\boldsymbol{k}}}-N_{-, \overline{\boldsymbol{k}}}\right|}{2}, \quad \text { with } \\
\left|N_{+, \overline{\boldsymbol{k}}}-N_{-, \overline{\boldsymbol{k}}}\right| \equiv & \max \left\{\left|N_{+,\left(k_{x}, 0\right)}-N_{-,\left(k_{x}, 0\right)}\right|,\right. \\
\left.\left|N_{+,\left(k_{x}, \pi\right)}-N_{-,\left(k_{x}, \pi\right)}\right|\right\}, & \left|N_{-, \overline{\boldsymbol{k}}_{c}}\right| \equiv \min \left\{\left|N_{+,\left(k_{x}, 0\right)}-N_{-,\left(k_{x}, 0\right)}\right|,\right. \\
& \left.\left|N_{+,\left(k_{x}, \pi\right)}-N_{-,\left(k_{x}, \pi\right)}\right|\right\} .
\end{aligned}
$$

Mapping.-Suppose that a screw-symmetric crystal is characterized by the four quantities $\left\{N_{ \pm \bar{\xi}, \bar{k}}, N_{ \pm, \overline{\boldsymbol{k}}_{c}}\right\}$ at a particular $k_{x}$. The eigenspectrum of the Wilson loop $\mathcal{W}[l]$, with $l$ parametrized by fixed $k_{x}$ and $k_{y} \in[0,2 \pi)$, consists of (i) eigenvalue $-\bar{\xi}$ with multiplicity $\left(N_{+, \bar{k}_{c}}-N_{\bar{\xi}, \bar{k}}\right)$, (ii) eigenvalue $+\bar{\xi}$ with multiplicity $\left(N_{-, \bar{k}_{c}}-N_{\bar{\xi}, \bar{k}}\right)$, and (iii) $N_{\bar{\xi}, \bar{k}}$ pairs of complex-conjugate eigenvalues.

In the above examples, the $\mathcal{W}$ spectrum of (a) comprises one +1 and one -1 eigenvalue; for (b), there are one +1 eigenvalue, one -1 eigenvalue, and one complex-conjugate pair; (c) has one complex-conjugate pair only. For a screw-symmetric crystal with filling two and four, we tabulate the possible mappings, for $k_{x}=0$, in Tables I and II, respectively.

It is useful to connect this mapping to the notation $W_{ \pm}\left(k_{x}\right)$, which we define in Sec. III C as the number of robust $\pm 1 \mathcal{W}$ eigenvalues; i.e.,

$$
W_{ \pm}\left(k_{x}\right)=N_{\mp \operatorname{sign}[\bar{\xi}], \bar{k}_{c}}-N_{\bar{\xi}, \bar{k}} .
$$

Now we finish our proof of Eq. (A1), which relates $W_{ \pm}\left(k_{x}\right)$ to the minimal numbers of Dirac crossings along the screw lines. First,

$$
\begin{aligned}
2 \max & \left\{W_{+}\left(k_{x}\right), W_{-}\left(k_{x}\right)\right\} \\
& =2 \max \left\{N_{+, \overline{\boldsymbol{k}}_{c}}-N_{\left.\bar{\xi}_{\bar{\xi}, \bar{k}}, N_{-, \overline{\boldsymbol{k}}_{c}}-N_{\bar{\xi}, \overline{\boldsymbol{k}}}\right\}}\right. \\
& =2 \max \left\{N_{+, \overline{\boldsymbol{k}}_{c}}, N_{-, \overline{\boldsymbol{k}}_{c}}\right\}-2 N_{\bar{\xi}, \overline{\boldsymbol{k}}} \\
& =2 \max \left\{N_{+, \overline{\boldsymbol{k}}_{c}}, N_{-, \overline{\boldsymbol{k}}_{c}}\right\}-f+\left|N_{+, \overline{\boldsymbol{k}}}-N_{-, \overline{\boldsymbol{k}}}\right| \\
& =2 \max \left\{N_{+, \overline{\boldsymbol{k}}_{c}}, N_{-, \overline{\boldsymbol{k}}_{c}}\right\}-N_{+, \overline{\boldsymbol{k}}_{c}}-N_{-, \overline{\boldsymbol{k}}_{c}}+\left|N_{+, \overline{\boldsymbol{k}}}-N_{-, \overline{\boldsymbol{k}}}\right| \\
& =\left|N_{+, \overline{\boldsymbol{k}}_{c}}-N_{-, \overline{\boldsymbol{k}}_{c}}\right|+\left|N_{+, \overline{\boldsymbol{k}}}-N_{-, \overline{\boldsymbol{k}}}\right| \\
& =\left|N_{+,\left(k_{x}, 0\right)}-N_{-,\left(k_{x}, 0\right)}\right|+\left|N_{+,\left(k_{x}, \pi\right)}-N_{-,\left(k_{x}, \pi\right)}\right|,
\end{aligned}
$$

which, upon substitution of Eq. (1) with $k_{x}=0$, leads immediately to the first line of Eq. (A1). In the first equality of Eq. (A12) we apply (i) and (ii) in the above mapping; in the third equality we apply Eq. (A10); in the fourth, $f=N_{+, \overline{\boldsymbol{k}}_{c}}+N_{-, \overline{\boldsymbol{k}}_{c}}$. Similarly,
TABLE II. Topological characterization of a crystal with filling $f=4$ and either glide or screw symmetry. The notation used here is explained in the caption of Table I.

\begin{tabular}{lcccc}
\hline \hline$N_{+, \boldsymbol{k}_{1}}$ & $N_{+, \boldsymbol{k}_{2}}$ & $\mathcal{W}\left(k_{x}\right)$ & $\bar{D}_{l\left(k_{x}\right)}$ & $D_{\boldsymbol{k}_{1}}-D_{\boldsymbol{k}_{2}} \bmod 2$ \\
\hline 2 & 2 & {$\left[\lambda_{1} \lambda_{1}^{*} \lambda_{2} \lambda_{2}^{*}\right]$} & 0 & 0 \\
3 & 2 & {$\left[\lambda_{1} \lambda_{1}^{*}+-\right]$} & 1 & 1 \\
3 & 3 & {$\left[\lambda_{1} \lambda_{1}^{*}++\right]$} & 2 & 0 \\
3 & 1 & {$\left[\lambda_{1} \lambda_{1}^{*}--\right]$} & 2 & 0 \\
4 & 2 & {$[++--]$} & 2 & 0 \\
4 & 3 & {$[+++-]$} & 3 & 1 \\
4 & 1 & {$[+---]$} & 3 & 1 \\
4 & 0 & {$[----]$} & 4 & 0 \\
4 & 4 & {$[++++]$} & 4 & 0 \\
\hline \hline
\end{tabular}

$$
\begin{aligned}
2 & \min \left\{W_{+}\left(k_{x}\right), W_{-}\left(k_{x}\right)\right\} \\
& =2 \min \left\{N_{+, \overline{\boldsymbol{k}}_{c}}, N_{-, \overline{\boldsymbol{k}}_{c}}\right\}-N_{+, \overline{\boldsymbol{k}}_{c}}-N_{-, \overline{\boldsymbol{k}}_{c}}+\left|N_{+, \overline{\boldsymbol{k}}}-N_{-, \overline{\boldsymbol{k}}}\right| \\
& =-\left|N_{+, \overline{\boldsymbol{k}}_{c}}-N_{-, \overline{\boldsymbol{k}}_{c}}\right|+\left|N_{+, \overline{\boldsymbol{k}}}-N_{-, \bar{k}}\right| \\
& =|-| N_{+, \bar{k}_{c}}-N_{-, \overline{\boldsymbol{k}}_{c}}|+| N_{+, \overline{\boldsymbol{k}}}-N_{-, \bar{k}}|| \\
& =|| N_{+,\left(k_{x}, 0\right)}-N_{-,\left(k_{x}, 0\right)}|-| N_{+,\left(k_{x}, \pi\right)}-N_{-,\left(k_{x}, \pi\right)}||
\end{aligned}
$$

leads to the second line of Eq. (A1).

We remark that a glide reflection, composed of a reflection $(y \rightarrow-y)$ and a half-lattice translation along $\boldsymbol{e}_{x}$, also maps the wave vector $\left(k_{x}, k_{y}\right) \rightarrow\left(k_{x},-k_{y}\right)$, and is represented by a unitary matrix (squaring to identity) multiplied by a phase factor $\exp \left(-i k_{x} / 2\right)$, just as for a screw rotation in Eq. (A6). Consequently, our analysis in this section is also applicable to glide reflections if we cosmetically substitute "screw eigenvalue" with "glide eigenvalue."

\section{APPENDIX B: NONSYMMORPHIC CRITERION FOR THE $\mathbb{Z}_{2}$-TOPOLOGICAL PHASE}

To prove our criterion for the gapped, $\mathbb{Z}_{2}$-topological phase in Sec. III D, we employ the Fu-Kane obstruction formulation [52] of the $\mathbb{Z}_{2}$ invariant:

$$
\nu \sim \frac{i}{2 \pi}\left[-\int_{\partial \tau_{1 / 2}} \operatorname{Tr}[\boldsymbol{A}(\boldsymbol{k})] \cdot d \boldsymbol{k}+\int_{\tau_{1 / 2}} F(\boldsymbol{k}) d^{2} \boldsymbol{k}\right],
$$

where $\sim$ denotes equality modulo $2, \tau_{1 / 2}$ denotes the half Brillouin zone illustrated in Fig. 5, $\partial \tau_{1 / 2}$ denotes the oriented boundary of $\tau_{1 / 2}$ (blue line with arrows in the same figure), $\operatorname{Tr}[\boldsymbol{A}]$ denotes the $U(1)$ Berry connection

$$
\operatorname{Tr}[\boldsymbol{A}(\boldsymbol{k})]=\sum_{i=1}^{f}\left\langle u_{i, \boldsymbol{k}} \mid \nabla_{\boldsymbol{k}} u_{i, \boldsymbol{k}}\right\rangle,
$$

and $F$ denotes the U(1) Berry curvature 


$$
F(\boldsymbol{k})=\partial_{k_{x}} \operatorname{Tr}\left[A_{y}(\boldsymbol{k})\right]-\partial_{k_{y}} \operatorname{Tr}\left[A_{x}(\boldsymbol{k})\right]
$$

In the $\mathbb{Z}_{2}$ classification, even (odd) $\nu$ identifies a trivial (topological) phase. Implicit in the definition of $\nu$ is our use of the time-reversal-symmetric gauge, which constrains wave functions related by time reversal $T$. Specifically, we divide the number of filled bands, $2 f$ (counting both spin components), into $f$ pairs (labeled by an index $\alpha$ ), such that each pair is Kramers degenerate at the inversion-invariant momenta. Each Kramers pair of bands is further labeled by the index $I, I I$, and is constrained as

$$
\begin{aligned}
\left|u_{I, \alpha,-k}\right\rangle & =T\left|u_{I I, \alpha, k}\right\rangle, \\
\left|u_{I I, \alpha,-k}\right\rangle & =-T\left|u_{I, \alpha, k}\right\rangle,
\end{aligned}
$$

for all $\alpha \in\{1, \ldots, f\}$ and $\boldsymbol{k} \in \tau_{1 / 2}$. In the remainder of this appendix, our goal is to derive

$$
\nu=\frac{i}{\pi} \ln \operatorname{det}\left[\mathcal{W}_{\uparrow}(0)\right] \bmod 2,
$$

where $\mathcal{W}_{\uparrow}(0)$ is the Wilson loop of the $f$ filled bands for a single spin component in the spin-orbit-free metal. Equivalently, $\nu$ is odd (corresponding to the $\mathbb{Z}_{2}$-topological phase) if and only if $\operatorname{det}\left[\mathcal{W}_{\uparrow}(0)\right]=-1$, which restates criterion (ii) in Sec. III D. Having previously proven the equivalence of criteria (i)-(iii) in Sec. III D, our claim then follows.

\section{Proof of Eq. (B5)}

The nonsymmorphic symmetry (whether screw $\bar{C}_{2 x}$ or glide $\left.\bar{M}_{y}\right)$ implies $F\left(k_{x}, k_{y}\right)=-F\left(k_{x},-k_{y}\right)$ and thus that its integral over $\tau_{1 / 2}$ in Eq. (B1) vanishes. What remains in Eq. (B1) is the line integral of $\operatorname{Tr}[\boldsymbol{A}(\boldsymbol{k})]$ over the oriented loop $\partial \tau_{1 / 2}$. A convenient choice consistent with Eq. (B4) is a periodic gauge $\boldsymbol{A}\left(k_{x}, k_{y}\right)=\boldsymbol{A}\left(k_{x}, k_{y}+2 \pi\right)$. Then the line integral over $\partial \tau_{1 / 2}$ reduces to

$\nu \sim-\frac{i}{2 \pi}\left[\int_{l(0)} \operatorname{Tr}[\boldsymbol{A}(\boldsymbol{k})] \cdot d \boldsymbol{k}-\int_{l(\pi)} \operatorname{Tr}[\boldsymbol{A}(\boldsymbol{k})] \cdot d \boldsymbol{k}\right]$,

where $l\left(k_{x}\right)$ are the oriented, constant- $k_{x}$ loops illustrated in Fig. 2(c). Taking advantage of the time-reversalsymmetric gauge Eq. (B4), we decompose the U(1) Berry connection as

$$
\operatorname{Tr}[\boldsymbol{A}(\boldsymbol{k})]=\boldsymbol{A}_{I}(\boldsymbol{k})+\boldsymbol{A}_{I I}(\boldsymbol{k}),
$$

with

$$
\boldsymbol{A}_{s}(\boldsymbol{k})=\sum_{\alpha=1}^{f}\left\langle u_{s, \alpha, k} \mid \nabla_{\boldsymbol{k}} u_{s, \alpha, \boldsymbol{k}}\right\rangle, \quad \text { for } s \in\{I, I I\},
$$

satisfying

$$
\boldsymbol{A}_{I}(-\boldsymbol{k})=\boldsymbol{A}_{I I}(\boldsymbol{k})
$$

This leads to

$$
\nu \sim-\frac{i}{\pi}\left[\int_{l(0)} A_{I}(k) \cdot d \boldsymbol{k}-\int_{l(\pi)} A_{I}(k) \cdot d \boldsymbol{k}\right] .
$$

Since any gauge transformation that preserves Eq. (B4) must maintain the decomposition given in Eq. (B7), $U(f)$ gauge transformations within the Kramers subspace $I$ are allowed, leading to

$$
\int_{l^{\prime}} \boldsymbol{A}_{I}(\boldsymbol{k}) \cdot d \boldsymbol{k} \rightarrow \int_{l^{\prime}} \boldsymbol{A}_{I}(\boldsymbol{k}) \cdot d \boldsymbol{k}+i 2 \pi n,
$$

with $n \in \mathbb{Z}$. The subspace $I I$ transforms dependently to maintain Eq. (B9); therefore, $\nu$ is gauge invariant modulo 2 [112]. If we had not imposed the time-reversal-symmetric gauge, it is well known that any loop integral of the $\mathrm{U}(1)$ connection [exemplified by Eq. (B6)] would only be gauge invariant modulo 1 .

Suppose an interpolation (parametrized by $z \in[0,1]$ ) exists between metallic $(z=0)$ and gapped $(z=1)$ phases, which preserves both time-reversal and nonsymmorphic symmetries, as well as the spectral gap along both $l(0)$ and $l(\pi)$. We then introduce $\boldsymbol{A}(\boldsymbol{k}) \rightarrow \boldsymbol{A}(\boldsymbol{k} ; z)$ to label the connection at a particular point in the interpolation. We would like to show that two polarization quantities, defined by

$$
\begin{aligned}
\mathcal{P}\left(l^{\prime} ; z\right) & \equiv \frac{i}{2 \pi} \int_{l^{\prime}} \operatorname{Tr}[\boldsymbol{A}(\boldsymbol{k} ; z)] \cdot d \boldsymbol{k}, \\
l^{\prime} & =l(0), l(\pi),
\end{aligned}
$$

in the time-reversal-symmetric gauge, are invariant modulo 2 throughout this interpolation. Having shown this, we would conclude from Eq. (B6) that $\nu$, the $\mathbb{Z}_{2}$ invariant in the fully gapped, spin-orbit-coupled phase, obeys

$$
\nu \sim \mathcal{P}(l(\pi) ; 0)-\mathcal{P}(l(0) ; 0),
$$

which we evaluate with wave functions of the spin-orbitfree metal.

Proof of invariance.-Since $l^{\prime}$ is mapped onto $-l^{\prime}$ by the nonsymmorphic symmetry, Eqs. (3) and (4) show that $\mathcal{P}\left(l^{\prime} ; z\right)$ is independent of $z$, modulo large gauge transformations that modify $\mathcal{P}$ by some additive integer. The allowed gauge transformations that preserve Eq. (B4) cannot add an even integer to $\mathcal{P}$, as we showed earlier in this appendix.

In the spin-orbit-free limit, we may identify the Kramers indices ( $I$ and $I I$ ) with the two spin components ( $\uparrow$ and $\downarrow$ ), for an arbitrarily chosen spin quantization axis. Additionally, using the time-reversal-symmetric gauge, we express Eq. (B13) as

$$
\nu \sim-\frac{i}{\pi}\left[\int_{l(0)} \boldsymbol{A}_{\uparrow}(\boldsymbol{k}) \cdot d \boldsymbol{k}-\int_{l(\pi)} \boldsymbol{A}_{\uparrow}(\boldsymbol{k}) \cdot d \boldsymbol{k}\right],
$$


just as we do in Eq. (B10). Now applying the identity Eq. (3) with $\mathcal{W}\left[l\left(k_{x}\right)\right] \equiv \mathcal{W}\left(k_{x}\right)$, and further adding the subscript $\mathcal{W} \rightarrow \mathcal{W}_{\uparrow}$ to remind ourselves of the spin projection,

$$
\nu \sim \frac{i}{\pi}\left\{\ln \operatorname{det}\left[\mathcal{W}_{\uparrow}(0)\right]-\ln \operatorname{det}\left[\mathcal{W}_{\uparrow}(\pi)\right]\right\} .
$$

As described in Sec. III B, the oriented loop $l(\pi)$ intersects the screw-invariant (or glide-invariant) points $X$ and $M$, where, at each of $X$ and $M$, the nonsymmorphic eigenvalues of filled states always comprise $\pm i$ pairs. From the mapping of Appendix A, we deduce that all eigenvalues of $\mathcal{W}_{\uparrow}(\pi)$ come in complex-conjugate pairs, i.e., $\operatorname{det}\left[\mathcal{W}_{\uparrow}(\pi)\right]=+1$, leading us finally to Eq. (B5).

\section{APPENDIX C: DERIVATION OF THE TIGHT-BINDING MODEL OF MONOLAYER $M X_{2}$}

To obtain a minimal, tight-binding model of the $M X_{2}$ compounds considered in this paper, we perform a Wannier interpolation of the four bands closest to the Fermi level [60]. The Wannier functions thus obtained transform as $d_{x^{2}-y^{2}}$ orbitals centered close to the $M$ atoms and $p_{x}$-type orbitals centered close to the $X-1$ atoms, as plotted in Fig. 10 and tabulated in Table III. We remark that each Wyckoff position in the symmetry group has a fixed coordinate in $\boldsymbol{e}_{x}$ (corresponding to the atomic position $x / a= \pm 0.25$ ) but not in $\boldsymbol{e}_{y}$; consequently, the centers of the Wannier functions are slightly displaced in $\boldsymbol{e}_{y}$ from the atomic centers.

Let us consider all symmetry-allowed, nearest-neighbor hoppings and, additionally, two, next-nearest-neighbor hoppings (denoted by $t_{d}$ and $t_{p}$ ) along the chain where atoms are closely spaced; these hoppings are illustrated in Fig. 10. In a basis of real Wannier functions, time-reversal symmetry constrains all hopping parameters to be real. $\bar{M}_{x}$ transforms the creation operators as

$$
\begin{aligned}
& \bar{M}_{x} a_{\ell,\left[R_{x}, R_{y}\right]}^{\dagger} \bar{M}_{x}^{-1}=(-1)^{l} a_{\ell,\left[-R_{x}, R_{y}\right]}^{\dagger}, \\
& \bar{M}_{x} b_{\ell,\left[R_{x}, R_{y}\right]}^{\dagger} \bar{M}_{x}^{-1}=(-1)^{l} b_{\ell,\left[-R_{x}+a, R_{y}\right]}^{\dagger},
\end{aligned}
$$

where $l=1$ for $\ell=p$ and $l=0$ for $\ell=d$. This suppresses nearest-neighbor hopping terms of the form $t_{p d}^{A A} a_{p, \boldsymbol{R}}^{\dagger} a_{d, \boldsymbol{R}}$ and $t_{p d}^{B B} b_{p, \boldsymbol{R}}^{\dagger} b_{d, \boldsymbol{R}}$. Given our choice of unit cell, intracell hoppings of the form $t_{\ell, \ell^{\prime}}^{A B} a_{\ell, \boldsymbol{R}}^{\dagger} b_{\ell^{\prime}, \boldsymbol{R}}$ are mapped onto $(-1)^{1-\delta_{\ell, \ell^{\prime}}} t_{\ell \ell^{\prime}}^{A B} a_{\ell,\left[-R_{x}, R_{y}\right]}^{\dagger} b_{\ell^{\prime},\left[-R_{x}+a, R_{y}\right]}$, which corresponds to an intercell hopping from the neighboring cell in the $+\boldsymbol{e}_{x}$ direction. For $\ell \neq \ell^{\prime}$, this hopping acquires a minus sign under $\bar{M}_{x}$, which accounts for the factor of $(-1)^{n}$ in the Hamiltonian. Spatial inversion transforms the creation operators as (a)

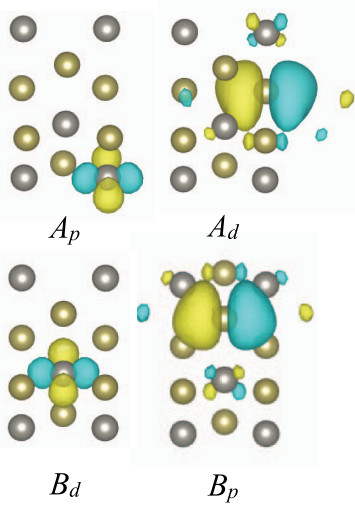

(b)

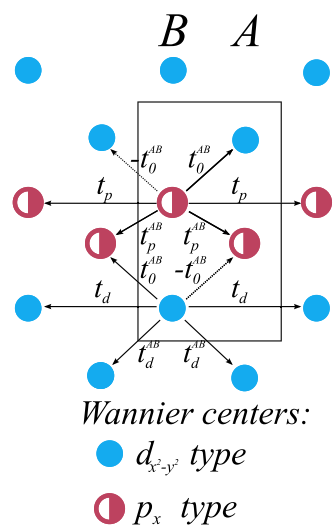

FIG. 10. (a) Plots of the Wannier functions obtained by a 4band Wannier interpolation. The functions are labeled by their orbital character $\ell=d, p$ and their sublattice index $A, B$. Gray spheres represent $M$ atoms, whereas as ochre spheres represent $X$ atoms. (b) Symmetry-allowed hoppings considered for the tightbinding model. All hoppings are nearest-neighbor hoppings with the exception of the intrasublattice hoppings $t_{d}$ and $t_{p}$. All symbols below the letter A (B) belong to the A (B) sublattice.

$$
\begin{aligned}
& \mathcal{I} a_{\ell, \boldsymbol{R}}^{\dagger} \mathcal{I}^{-1}=(-1)^{l} b_{\ell,-\boldsymbol{R}}^{\dagger}, \\
& \mathcal{I} b_{\ell, \boldsymbol{R}}^{\dagger} \mathcal{I}^{-1}=(-1)^{l} a_{\ell,-\boldsymbol{R}}^{\dagger},
\end{aligned}
$$

which enforces $t_{\ell, \ell^{\prime}}^{A B}=-t_{\ell, \ell^{\prime}}^{B A}$ for the interchain hoppings in the same cell with $\ell \neq \ell^{\prime}$.

In summary, the symmetry-allowed nearest-neighbor hoppings are the intrachain hoppings $t_{\ell, \ell}^{A B} \equiv t_{\ell}^{A B}$ with $\ell=d, p$. The symmetry-allowed interchain hoppings

TABLE III. Wannier function centers and atomic positions for $\mathrm{WTe}_{2}$ (left). Hopping parameters obtained from the Wannier interpolation (right). The positions are given in units of the lattice vectors (the origin is the center of inversion), while the hopping parameters are given in $\mathrm{eV}$.

\begin{tabular}{lrr}
\hline \hline Wannier center/Atomic position & $x / a$ & $y / b$ \\
\hline$A_{d}$ & -0.25 & 0.35 \\
$B_{d}$ & 0.25 & -0.35 \\
$A_{p}$ & -0.25 & -0.11 \\
$B_{p}$ & 0.25 & 0.11 \\
$\mathrm{~W}$ & 0.25 & -0.32 \\
$\mathrm{Te}_{1}$ & 0.25 & 0.42 \\
$\mathrm{Te}_{2}$ & 0.25 & 0.07 \\
\hline \hline
\end{tabular}

\section{Hopping/eV}

\begin{tabular}{lr}
\hline$\mu_{d}$ & 1.44 \\
$\mu_{p}$ & -0.38 \\
$t_{d}$ & -0.28 \\
$t_{p}$ & 0.93 \\
$t_{d}^{A B}$ & 0.52 \\
$t_{p}^{A B}$ & 0.40 \\
$t_{0}^{A B}$ & 1.02 \\
\hline \hline
\end{tabular}


are $t_{d, p}^{A B} \equiv t_{0}^{A B}$. The latter switch sign depending on the hopping direction due to $\bar{M}_{x}$ and $\mathcal{I}$ [see Fig. 10(b)]. The real-space Hamiltonian then reads:

$$
\begin{aligned}
H= & \sum_{\boldsymbol{R}}\left[\sum_{\ell} \mu_{\ell}\left(a_{\ell, \boldsymbol{R}}^{\dagger} a_{\ell, \boldsymbol{R}}+b_{\ell, \boldsymbol{R}}^{\dagger} b_{\ell, \boldsymbol{R}}\right)\right. \\
& +\sum_{\ell} t_{\ell}\left(a_{\ell, \boldsymbol{R}+\boldsymbol{e}_{x}}^{\dagger} a_{\ell, \boldsymbol{R}}+b_{\ell, \boldsymbol{R}+\boldsymbol{e}_{x}}^{\dagger} b_{\ell, \boldsymbol{R}}\right) \\
& +\sum_{n=0}^{1}(-1)^{n} t_{0}^{A B}\left(b_{p, \boldsymbol{R}+n e_{x}}^{\dagger} a_{d, \boldsymbol{R}}-b_{d, \boldsymbol{R}+n e_{x}}^{\dagger} a_{p, \boldsymbol{R}}\right) \\
& \left.+\sum_{\ell} \sum_{\boldsymbol{\delta}_{\ell}} t_{l}^{A B} a_{\ell, \boldsymbol{R}+\boldsymbol{\delta}_{\ell}}^{\dagger} b_{\ell, \boldsymbol{R}}\right]+ \text { H.c. }
\end{aligned}
$$

We construct a basis of Bloch waves by the Fourier transformation

$$
\begin{aligned}
c_{\boldsymbol{k}, \ell, A}^{\dagger} & =\frac{1}{\sqrt{N}} \sum_{\boldsymbol{R}} e^{i \boldsymbol{k} \cdot\left(\boldsymbol{R}+\boldsymbol{r}_{A, \ell}\right)} a_{\ell, \boldsymbol{R}}^{\dagger}, \\
c_{\boldsymbol{k}, \ell, B}^{\dagger} & =\frac{1}{\sqrt{N}} \sum_{\boldsymbol{R}} e^{i \boldsymbol{k} \cdot\left(\boldsymbol{R}+\boldsymbol{r}_{B, \ell}\right)} b_{\ell, \boldsymbol{R}}^{\dagger},
\end{aligned}
$$

where $N$ is the number of unit cells and $\boldsymbol{r}_{s, \ell}$ denotes the position of the $\ell$-type Wannier center in sublattice $s$, as illustrated in Fig. 2(a). In the basis $\left[c_{\boldsymbol{k}, d, A}^{\dagger}, c_{\boldsymbol{k}, p, A}^{\dagger}, c_{\boldsymbol{k}, d, B}^{\dagger}\right.$, $\left.c_{\boldsymbol{k}, p, B}^{\dagger}\right]$, the Hamiltonian is represented by the matrix

$$
\mathcal{H}(\boldsymbol{k})=\left(\begin{array}{cccc}
\mu_{d}+2 t_{d} \cos \left(k_{x}\right) & 0 & t_{d}^{A B} e^{-i \boldsymbol{k} \cdot\left(\boldsymbol{r}_{B, d}-\boldsymbol{r}_{A, d}\right)}\left(e^{i k_{y}}+e^{i\left(k_{y}-k_{x}\right)}\right) & t_{0}^{A B} e^{-i \boldsymbol{k} \cdot\left(\boldsymbol{r}_{B, p}-\boldsymbol{r}_{A, d}\right)}\left(1-e^{-i k_{x}}\right) \\
0 & \mu_{p}+2 t_{p} \cos \left(k_{x}\right) & t_{0}^{A B} e^{-i \boldsymbol{k} \cdot\left(\boldsymbol{r}_{B, d}-\boldsymbol{r}_{A, p}\right)}\left(e^{-i k_{x}}-1\right) & t_{p}^{A B} e^{-i \boldsymbol{k} \cdot\left(\boldsymbol{r}_{B, p}-\boldsymbol{r}_{A, p}\right)}\left(1+e^{-i k_{x}}\right) \\
\text { c.c } & \text { c.c } & \mu_{d}+2 t_{d} \cos \left(k_{x}\right) & 0 \\
\text { c.c } & \text { c.c } & 0 & \mu_{p}+2 t_{p} \cos \left(k_{x}\right)
\end{array}\right) .
$$

The relevant parameters for the Hamiltonian of $\mathrm{WTe}_{2}$ obtained by the Wannier interpolation of the DFT band structure are given in Table III. The Dirac crossing in the DFT band structure is type II, but this is not reproduced by the tight-binding Hamiltonian Eq. (C5) with parameters obtained from the interpolation. This is due to the truncation of longer-ranged hoppings which would further tilt the Dirac cone. To retain a minimal tight-binding model that accounts for the type-II nature of the Dirac crossing, we renormalize the intrasublattice hoppings $t_{d}, t_{p}$ while leaving the other values untouched, which results in a tilted type-II Dirac cone. Figure 6(a) is obtained by setting $t_{d}=-0.4 \mathrm{eV}$ and $t_{p}=1.34 \mathrm{eV}$ with a Fermi energy $\varepsilon_{F}=1.47 \mathrm{eV}$.

\section{APPENDIX D: CIRCULAR DICHROISM}

To calculate the photoelectron intensity in Eq. (12) due to light with polarization vector $\lambda$, one needs the transition matrix elements of the momentum operator $\boldsymbol{p}$ between the initial and final states of the electron; i.e., $P_{ \pm}^{i f}(\boldsymbol{k})=\lambda_{ \pm} \cdot\langle f, \boldsymbol{k}|\boldsymbol{p}| i, \boldsymbol{k}\rangle$. Within the three-step model of photoemission, $|i, \boldsymbol{k}\rangle$ and $|f, \boldsymbol{k}\rangle$ are the initial and final Bloch state at wave vector $\boldsymbol{k}$, respectively. We assume that the polarization of the light is in the plane of the bilayer; i.e., $\lambda_{ \pm}=[1, \pm i, 0]$. The expression for the matrix element can be cast in the form

$$
\begin{aligned}
P_{ \pm}^{i f}(\boldsymbol{k}) & =\lambda_{ \pm} \cdot\langle f, \boldsymbol{k}|\boldsymbol{p}| i, \boldsymbol{k}\rangle \\
& =\lambda_{ \pm} \cdot \int d \boldsymbol{r} e^{-i \boldsymbol{k} r} u_{f k}(\boldsymbol{r})^{*} \boldsymbol{p} e^{i k \boldsymbol{r}} u_{i k}(\boldsymbol{r}) \\
& =\lambda_{ \pm} \cdot \int d \boldsymbol{r} e^{-i k \boldsymbol{r}} u_{f \boldsymbol{k}}(\boldsymbol{r})^{*}\left[\boldsymbol{k} e^{i k r} u_{i k}+e^{i k r} \boldsymbol{p} u_{i k}(\boldsymbol{r})\right] \\
& =\lambda_{ \pm} \cdot \int d \boldsymbol{r} u_{f k}(\boldsymbol{r})^{*} \boldsymbol{p} u_{i k} \boldsymbol{r} .
\end{aligned}
$$

The Fourier expansion of the periodic part of a Bloch state is

$$
u_{n k}(\boldsymbol{r})=\sum_{\boldsymbol{G}} c_{\boldsymbol{G k}}^{[n]} e^{i \boldsymbol{G r}}
$$

where $\boldsymbol{G}$ are reciprocal lattice vectors. One obtains

$$
P_{ \pm}^{i f}(\boldsymbol{k})=\boldsymbol{\lambda}_{ \pm} \cdot \sum_{\boldsymbol{G}} \boldsymbol{G} c_{\boldsymbol{G} \boldsymbol{k}}^{[f] *} c_{\boldsymbol{G} \boldsymbol{k}}^{[i]}
$$

A final state with only one nonzero coefficient $c_{\boldsymbol{G} k}^{f}$ leads to a vanishing $\mathrm{CD}$ signal, because

$$
\begin{aligned}
\mid P_{+}^{i f} & \left.(\boldsymbol{k})\right|^{2}-\left|P_{-}^{i f}(\boldsymbol{k})\right|^{2} \\
& =\left(\left|G_{x}+i G_{y}\right|^{2}-\left|G_{x}-i G_{y}\right|^{2}\right)\left|c_{\boldsymbol{G} \boldsymbol{k}}^{[f] *} c_{\boldsymbol{G} \boldsymbol{k}}^{[i]}\right|^{2} \\
& =0
\end{aligned}
$$


where $G_{x}, G_{y}$ are the components of $\boldsymbol{G}[113,114]$. Therefore, to obtain a nonzero $\mathrm{CD}$ signal one needs to use a final state with at least two nonzero plane-wave coefficients. It is reasonable to assume that the high-energy final Bloch states have only few nonzero plane-wave components, and we thus consider final states having nonzero coefficients $c_{G k}^{f}$ only for the four smallest in-plane $G$ vectors; i.e., $\boldsymbol{G}=[ \pm(2 \pi / a), 0,0],[0, \pm(2 \pi / b), 0]$. In addition, we assume that the final state at $k_{x}=0$ transforms as the even representation of $\bar{M}_{x}$, which is the only point symmetry present in the bilayer, and further assume that the periodic part of the final state does not depend on $\boldsymbol{k}$ and set the four nonzero coefficients to have the same value. These assumptions produce the calculated dichroic signals $D_{s}\left(\boldsymbol{k}, \varepsilon_{F}\right)$ we report in Sec. V B. Notice that we could have equally well assumed that the state at $k_{x}=0$ transformed as the odd representation of $\bar{M}_{x}$, but that would not have changed our qualitative conclusions regarding the dichroism in the bilayer.

The coefficients of the initial state are obtained using the plane-wave pseudopotential code in the QUANTUM ESPRESSO package. For Te and W, we use the pseudopotentials Te.pbe-hgh.UPF and W.pbe-hgh.UPF from the QUANTUM ESPRESSO database, respectively. The planewave cutoff is set to $80 \mathrm{Ry}$ with a $15 \times 8 \times 1 k$-point mesh in the BZ. The plane-wave coefficients are computed on a $25 \times 16$ mesh around the pockets [115].

[1] V. L. Ginzburg and L. D. Landau, On the Theory of Superconductivity, Zh. Eksp. Teor. Fiz. 20, 1064 (1950).

[2] J. Zak, Berry's Phase for Energy Bands in Solids, Phys. Rev. Lett. 62, 2747 (1989).

[3] F. D. M. Haldane, Model for a Quantum Hall Effect without Landau Levels: Condensed-Matter Realization of the "Parity Anomaly", Phys. Rev. Lett. 61, 2015 (1988).

[4] C. L. Kane and E. J. Mele, $Z_{2}$ Topological Order and the Quantum Spin Hall Effect, Phys. Rev. Lett. 95, 146802 (2005).

[5] A. Alexandradinata, Z. Wang, and B. A. Bernevig, Topological Insulators from Group Cohomology, Phys. Rev. X 6, 021008 (2016).

[6] M. V. Berry, Quantal Phase Factors Accompanying Adiabatic Changes, Proc. R. Soc. A 392, 45 (1984).

[7] M. Lax, Symmetry Principles in Solid State and Molecular Physics (Wiley-Interscience, New York, 1974).

[8] L. Michel and J. Zak, Connectivity of Energy Bands in Crystals, Phys. Rev. B 59, 5998 (1999).

[9] S. M. Young and C. L. Kane, Dirac Semimetals in Two Dimensions, Phys. Rev. Lett. 115, 126803 (2015).

[10] L. Michel and J. Zak, Elementary Energy Bands in Crystalline Solids, Europhys. Lett. 50, 519 (2000).

[11] Z. Song, S. M. Nie, H. Weng, and Z. Fang, Quantum Spin Hall State on Square-like Lattice, arXiv:1508.05220.

[12] S. M. Nie, Z. Song, H. Weng, and Z. Fang, Quantum Spin Hall Effect in Two-Dimensional Transition-Metal
Dichalcogenide Haeckelites, Phys. Rev. B 91, 235434 (2015).

[13] Y. Sun, C. Felser, and B. Yan, Graphene-like Dirac States and Quantum Spin Hall Insulators in Square-Octagonal $M X_{2}(M=\mathrm{Mo}, W ; X=\mathrm{S}, \mathrm{Se}, \mathrm{Te})$ Isomers, Phys. Rev. B 92, 165421 (2015).

[14] X. Wan, A. M. Turner, A. Vishwanath, and S. Y. Savrasov, Topological Semimetal and Fermi-Arc Surface States in the Electronic Structure of Pyrochlore Iridates, Phys. Rev. B 83, 205101 (2011).

[15] A. Alexandradinata, X. Dai, and B. A. Bernevig, WilsonLoop Characterization of Inversion-Symmetric Topological Insulators, Phys. Rev. B 89, 155114 (2014).

[16] R. Yu, X. L. Qi, A. Bernevig, Z. Fang, and X. Dai, Equivalent Expression of $Z_{2}$ Topological Invariant for Band Insulators Using the non-Abelian Berry Connection, Phys. Rev. B 84, 075119 (2011).

[17] A. A. Soluyanov and D. Vanderbilt, Wannier Representation of $Z_{2}$ Topological Insulators, Phys. Rev. B 83, 035108 (2011).

[18] M. Taherinejad, K. F. Garrity, and D. Vanderbilt, Wannier Center Sheets in Topological Insulators, Phys. Rev. B 89, 115102 (2014).

[19] A. Alexandradinata and B.A. Bernevig, Berry-Phase Description of Topological Crystalline Insulators, Phys. Rev. B 93, 205104 (2016).

[20] A. A. Soluyanov, D. Gresch, Z. Wang, Q. Wu, M. Troyer, X. Dai, and B.A. Bernevig, Type-II Weyl Semimetals, Nature (London) 527, 495 (2015).

[21] L. Huang et al., Spectroscopic Evidence for Type II Weyl Semimetal State in $\mathrm{MoTe}_{2}$, arXiv:1603.06482.

[22] K. Deng et al., Experimental Observation of Topological Fermi Arcs in Type-II Weyl Semimetal $\mathrm{MoTe}_{2}$, arXiv: 1603.08508 [Nat. Phys. (in press)].

[23] J. Jiang et al., Observation of the Type-II Weyl Semimetal Phase in $\mathrm{MoTe}_{2}$, arXiv:1604.00139.

[24] S. Katayama, A. Kobayashi, and Y. Suzumura, PressureInduced Zero-Gap Semiconducting State in Organic Conductor $\alpha$-(BEDT-TTF $)_{2} \mathrm{I}_{3}$ Salt, J. Phys. Soc. Jpn. 75, 054705 (2006).

[25] T. Kawarabayashi, Y. Hatsugai, T. Morimoto, and H. Aoki, Generalized Chiral Symmetry and Stability of Zero Modes for Tilted Dirac Cones, Phys. Rev. B 83, 153414 (2011).

[26] M. O. Goerbig, J.-N. Fuchs, G. Montambaux, and F. Piéchon, Tilted Anisotropic Dirac Cones in Quinoid-Type Graphene and $\alpha$-(BEDT-TTF $)_{2} \mathrm{I}_{3}$, Phys. Rev. B 78, 045415 (2008).

[27] M. Trescher, B. Sbierski, P. W. Brouwer, and E. J. Bergholtz, Quantum Transport in Dirac Materials: Signatures of Tilted and Anisotropic Dirac and Weyl Cones, Phys. Rev. B 91, 115135 (2015).

[28] E. J. Bergholtz, Z. Liu, M. Trescher, R. Moessner, and M. Udagawa, Topology and Interactions in a Frustrated Slab: Tuning from Weyl Semimetals to $\mathcal{C}>1$ Fractional Chern Insulators, Phys. Rev. Lett. 114, 016806 (2015).

[29] N. Harrison and S. E. Sebastian, Dirac Nodal Pockets in the Antiferromagnetic Parent Phase of FeAs Superconductors, Phys. Rev. B 80, 224512 (2009).

[30] G. A. H. Schober, H. Murakawa, M. S. Bahramy, R. Arita, Y. Kaneko, Y. Tokura, and N. Nagaosa, Mechanisms of 
Enhanced Orbital Dia- and Paramagnetism: Application to the Rashba Semiconductor BiTeI, Phys. Rev. Lett. 108, 247208 (2012).

[31] Y. Xu, F. Zhang, and C. Zhang, Structured Weyl Points in Spin-Orbit Coupled Fermionic Superfluids, Phys. Rev. Lett. 115, 265304 (2015).

[32] H. Isobe and N. Nagaosa, Coulomb Interaction Effect in Weyl Fermions with Tilted Energy Dispersion in Two Dimensions, Phys. Rev. Lett. 116, 116803 (2016).

[33] T. E. O'Brien, M. Diez, and C. W. J. Beenakker, Magnetic Breakdown and Klein Tunneling in a Type-II Weyl Semimetal, Phys. Rev. Lett. 116, 236401 (2016).

[34] Y. M. Jhon, Y. Kim, Y. I. Jhon, J. Park, J. H. Kim, and S. Lee, Anomalous Raman Scattering and Lattice Dynamics in Mono-and Few-Layer $\mathrm{WTe}_{2}$, Nanoscale 8, 2309 (2015).

[35] L. Wang, I. Gutiérrez-Lezama, C. Barreteau, N. Ubrig, E. Giannini, and A. F. Morpurgo, Tuning Magnetotransport in a Compensated Semimetal at the Atomic Scale, Nat. Commun. 6, 8892 (2015).

[36] M. N. Ali et al., Large, Non-Saturating Magnetoresistance in $\mathrm{WTe}_{2}$, Nature (London) 514, 205 (2014).

[37] M. N. Ali, L. Schoop, J. Xiong, S. Flynn, Q. Gibson, M. Hirschberger, N. P. Ong, and R. J. Cava, Correlation of Crystal Quality and Extreme Magnetoresistance of $\mathrm{WTe}_{2}$, Europhys. Lett. 110, 67002 (2015).

[38] J. Jiang et al., Signature of Strong Spin-Orbital Coupling in the Large Nonsaturating Magnetoresistance Material $\mathrm{WTe}_{2}$, Phys. Rev. Lett. 115, 166601 (2015).

[39] Y. Liu, G. Bian, T. Miller, and T.-C. Chiang, Visualizing Electronic Chirality and Berry Phases in Graphene Systems Using Photoemission with Circularly Polarized Light, Phys. Rev. Lett. 107, 166803 (2011).

[40] I. Gierz, M. Lindroos, H. Hochst, C. R. Ast, and K. Kern, Graphene Sublattice Symmetry and Isospin Determined by Circular Dichroism in Angle-Resolved Photoemission Spectroscopy, Nano Lett. 12, 3900 (2012).

[41] X. Qian, J. Liu, L. Fu, and J. Li, Quantum Spin Hall Effect in Two-Dimensional Transition Metal Dichalcogenides, Science 346, 1344 (2014).

[42] R. Roy, $Z_{2}$ Classification of Quantum Spin Hall Systems: An Approach Using Time-Reversal Invariance, Phys. Rev. B 79, 195321 (2009).

[43] B. A. Bernevig and S. C. Zhang, Quantum Spin Hall Effect, Phys. Rev. Lett. 96, 106802 (2006).

[44] A. Mar, S. Jobic, and J. A. Ibers, Metal-Metal vs Tellurium-Tellurium Bonding in $\mathrm{WTe}_{2}$ and Its Ternary Variants TaIrTe4 and NbIrTe4, J. Am. Chem. Soc. 114, 8963 (1992).

[45] J. D. Corbett and D. H. Guthrie, A Second Infinite-Chain Form of Zirconium Diiodide $(\beta)$ and Its Coherent Intergrowth with $\alpha$-Zirconium Diiodide, Inorg. Chem. 21, 1747 (1982).

[46] Z. Wang, D. Gresch, A. A. Soluyanov, W. Xie, S. Kushwaha, X Dai, M. Troyer, R. J. Cava, and B. A. Bernevig, MoTe $\mathrm{T}_{2}$ : Weyl and Line Node Topological Metal, Phys. Rev. Lett. 117, 056805 (2016).

[47] T. Michael, Group Theory and Quantum Mechanics (Dover, New York, 2003).
[48] Z. Wang, A. Alexandradinata, R. J. Cava, and B. A. Bernevig, Hourglass Fermions, Nature (London) 532, 189 (2016).

[49] L. Lu, J. D. Joannopoulos, and M. Soljačić, Topological Photonics, Nat. Photonics 8, 821 (2014).

[50] L. Lu, Z. Wang, D. Ye, L. Ran, L. Fu, J. D. Joannopoulos, and M. Soljačić, Experimental Observation of Weyl Points, Science 349, 622 (2015).

[51] F. Wilczek and A. Zee, Appearance of Gauge Structure in Simple Dynamical Systems, Phys. Rev. Lett. 52, 2111 (1984).

[52] L. Fu and C. L. Kane, Time Reversal Polarization and $a Z_{2}$ Adiabatic Spin Pump, Phys. Rev. B 74, 195312 (2006).

[53] R. D. King-Smith and D. Vanderbilt, Theory of Polarization of Crystalline Solids, Phys. Rev. B 47, 1651 (1993).

[54] D. Vanderbilt and R. D. King-Smith, Electric Polarization as a Bulk Quantity and Its Relation to Surface Charge, Phys. Rev. B 48, 4442 (1993).

[55] M. Shuichi, Phase Transition between the Quantum Spin Hall and Insulator Phases in 3D: Emergence of a Topological Gapless Phase, New J. Phys. 9, 356 (2007).

[56] Z. Wang, Y. Sun, X.-Q. Chen, C. Franchini, G. Xu, H. Weng, X. Dai, and Z. Fang, Dirac Semimetal and Topological Phase Transitions in $A_{3} \mathrm{Bi}(A=\mathrm{Na}, K$, Rb), Phys. Rev. B 85, 195320 (2012).

[57] A. Alexandradinata, Z. Wang, and B. A. Bernevig, Quantum Glide Hall Insulators, (to be published).

[58] C. L. Kane and E. J. Mele, Quantum Spin Hall Effect in Graphene, Phys. Rev. Lett. 95, 226801 (2005).

[59] L. Fu and C. L. Kane, Topological Insulators with Inversion Symmetry, Phys. Rev. B 76, 045302 (2007).

[60] A. A. Mostofi, J. R. Yates, Y.-S. Lee, I. Souza, D. Vanderbilt, and N. Marzari, Wannier90: A Tool for Obtaining Maximally-Localised Wannier Functions, Comput. Phys. Commun. 178, 685 (2008).

[61] G. E. Volovik, Lifshitz. Transitions via the Type-II Dirac and Type-II Weyl Points, arXiv:1604.00849.

[62] I Pletikosić, M. N. Ali, A. V. Fedorov, R. J. Cava, and T. Valla, Electronic Structure Basis for the Extraordinary Magnetoresistance in $\mathrm{WTe}_{2}$, Phys. Rev. Lett. 113, 216601 (2014).

[63] Z. Zhu, X. Lin, J. Liu, B. Fauque, Q. Tao, C. Yang, Y. Shi, and K. Behnia, Quantum Oscillations, Thermoelectric Coefficients and the Fermi Surface of Semi-Metallic $\mathrm{WTe}_{2}$, Phys. Rev. Lett. 114, 176601 (2015).

[64] G. Schönhense, Circular Dichroism and Spin Polarization in Photoemission from Adsorbates and Non-Magnetic Solids, Phys. Scr. T31, 255 (1990).

[65] S. Hüfner, Photoelectron Spectroscopy: Principles and Applications (Springer Science \& Business Media, New York, 2013), Vol. 82.

[66] M. Mulazzi, G. Rossi, J. Braun, J. Minár, H. Ebert, G. Panaccione, I. Vobornik, and J. Fujii, Understanding Intensities of Angle-Resolved Photoemission with Circularly Polarized Radiation from a Cu(111) Surface State, Phys. Rev. B 79, 165421 (2009).

[67] M. Ärrälä, J. Nieminen, J. Braun, H. Ebert, and M. Lindroos, Photon Energy Dependence of Circular Dichroism of the Au(111) Surface State, Phys. Rev. B 88, 195413 (2013). 
[68] M. R. Scholz et al., Reversal of the Circular Dichroism in Angle-Resolved Photoemission from $\mathrm{Bi}_{2} \mathrm{Te}_{3}$, Phys. Rev. Lett. 110, 216801 (2013).

[69] Z. K. Liu et al., A Stable Three-Dimensional Topological Dirac Semimetal $\mathrm{Cd}_{3} \mathrm{As}_{2}$, Nat. Mater. 13, 677 (2014).

[70] S. Borisenko, Q. Gibson, D. Evtushinsky, V. Zabolotnyy, B. Büchner, and R. J. Cava, Experimental Realization of a Three-Dimensional Dirac Semimetal, Phys. Rev. Lett. 113, 027603 (2014).

[71] M. Neupane et al., Observation of a Three-Dimensional Topological Dirac Semimetal Phase in High-Mobility $\mathrm{Cd}_{3} \mathrm{As}_{2}$, Nat. Commun. 5, 3786 (2014).

[72] Z. K. Liu et al., Discovery of a Three-Dimensional Topological Dirac Semimetal, $\mathrm{Na}_{3} \mathrm{Bi}$, Science 343, 864 (2014).

[73] S.-Y. Xu et al., Observation of Fermi Arc Surface States in a Topological Metal, Science 347, 294 (2015).

[74] H. Weng, C. Fang, Z. Fang, B. A. Bernevig, and X. Dai, Weyl Semimetal Phase in Noncentrosymmetric TransitionMetal Monophosphides, Phys. Rev. X 5, 011029 (2015).

[75] S.-M. Huang et al., A Weyl Fermion Semimetal with Surface Fermi Arcs in the Transition Metal Monopnictide TaAs Class, Nat. Commun. 6, 7373 (2015).

[76] B. Q. Lv et al., Experimental Discovery of Weyl Semimetal TaAs, Phys. Rev. X 5, 031013 (2015).

[77] B. Q. Lv et al., Observation of Weyl Nodes in TaAs, Nat. Phys. 11, 724 (2015).

[78] L.X. Yang et al., Weyl Semimetal Phase in the NonCentrosymmetric Compound TaAs, Nat. Phys. 11, 728 (2015).

[79] S.-Y. Xu et al., Discovery of a Weyl Fermion Semimetal and Topological Fermi Arcs, Science 349, 613 (2015).

[80] L. X. Yang et al., Weyl Semimetal Phase in the NonCentrosymmetric Compound TaAs, Nat. Phys. 11, 728 (2015).

[81] J. Liu and D. Vanderbilt, Weyl Semimetals from Noncentrosymmetric Topological Insulators, Phys. Rev. B 90, 155316 (2014).

[82] G. B. Halász and L. Balents, Time-Reversal Invariant Realization of the Weyl Semimetal Phase, Phys. Rev. B 85, 035103 (2012).

[83] D. Bulmash, C.-X. Liu, and X.-L. Qi, Prediction of a Weyl Semimetal in $\mathrm{Hg}_{1-x-y} \mathrm{Cd}_{x} \mathrm{Mn}_{y}$ Te, Phys. Rev. B 89, 081106 (2014).

[84] D. T. Son and B. Z. Spivak, Chiral Anomaly and Classical Negative Magnetoresistance of Weyl Metals, Phys. Rev. B 88, 104412 (2013).

[85] S. A. Parameswaran, T. Grover, D. A. Abanin, D. A. Pesin, and A. Vishwanath, Probing the Chiral Anomaly with Nonlocal Transport in Three-Dimensional Topological Semimetals, Phys. Rev. X 4, 031035 (2014).

[86] J. Xiong, S. K. Kushwaha, T. Liang, J. W. Krizan, M. Hirschberger, W. Wang, R. J. Cava, and N. P. Ong, Evidence for the Chiral Anomaly in the Dirac Semimetal $\mathrm{Na}_{3} \mathrm{Bi}$, Science 350, 413 (2015).

[87] S.-Y. Xu et al., Discovery of a Weyl Fermion State with Fermi Arcs in Niobium Arsenide, Nat. Phys. 11, 748 (2015).
[88] C. Shekhar et al., Extremely Large Magnetoresistance and Ultrahigh Mobility in the Topological Weyl Semimetal Candidate NbP, Nat. Phys. 11, 645 (2015).

[89] S.-Y. Xu et al., Experimental Discovery of a Topological Weyl Semimetal State in TaP, Sci. Adv. 1, e1501092 (2015).

[90] D. H. Keum et al., Bandgap Opening in Few-Layered Monoclinic $\mathrm{MoTe}_{2}$, Nat. Phys. 11, 482 (2015).

[91] Y. Qi et al., Superconductivity in Weyl Semimetal Candidate $\mathrm{MoTe}_{2}$, Nat. Commun. 7, 11038 (2016).

[92] X.-C. Pan et al., Pressure-Driven Dome-Shaped Superconductivity and Electronic Structural Evolution in Tungsten Ditelluride, Nat. Commun. 6, 7805 (2015).

[93] F. C. Chen et al., Superconductivity Enhancement in the S-Doped Weyl Semimetal Candidate $\mathrm{MoTe}_{2}$, Appl. Phys. Lett. 108, 162601 (2016).

[94] J. Zhou et al., Large-Area and High-Quality 2D Transition Metal Telluride, arXiv:1606.00126.

[95] J. M. Woods, J. Shen, P. Kumaravadivel, Y. Pang, Y. Xie, G. A. Pan, M. Li, E. I. Altman, L. Lu, and J. J. Cha, Suppression of Magnetoresistance in Thin $\mathrm{WTe}_{2}$ Flakes by Surface Oxidation, arXiv:1606.05756.

[96] C. Kittel, Introduction to Solid State Physics (Wiley, New York, 2004).

[97] F. F. Tafti, Q. D. Gibson, S. K. Kushwaha, N. Haldolaarachchige, and R. J. Cava, Resistivity Plateau and Extreme Magnetoresistance in LaSb, Nat. Phys. 12, 272 (2015).

[98] F. F. Tafti, Q. D. Gibson, S. K. Kushwaha, J. W. Krizan, N. Haldolaarachchige, and R. J. Cava, Temperature-Field Phase Diagram of Extreme Magnetoresistance in Lanthanum Monopnictides, arXiv:1602.01525.

[99] T. Liang, Q. Gibson, M. N. Ali, M. Liu, R. J. Cava, and N. P. Ong, Ultrahigh Mobility and Giant Magnetoresistance in the Dirac Semimetal Cd3As2, Nat. Mater. 14, 280 (2015).

[100] D. Hsieh, D. Qian, L. Wray, Y. Xia, Y. S. Hor, R. J. Cava, and M.Z. Hasan, A Topological Dirac Insulator in a Quantum Spin Hall Phase, Nature (London) 452, 970 (2008).

[101] T. H. Hsieh, H. Lin, J. Liu, W. Duan, A. Bansi, and L. Fu, Topological Crystalline Insulators in the SnTe Material Class, Nat. Commun. 3, 982 (2012).

[102] S.-Y. Xu et al., Observation of a TCI Phase and Topological Phase Transition in $\mathrm{Pb}_{1-x} \mathrm{Sn}_{x}$ Te, Nat. Commun. 3, 1192 (2012).

[103] Y. Tanaka, Z. Ren, T. Sato, K. Nakayama, S. Souma, T. Takahashi, K. Segawa, and Y. Ando, Experimental Realization of a Topological Crystalline Insulator in SnTe, Nat. Phys. 8, 800 (2012).

[104] N. Alidoust et al., A New Form of (Unexpected) Dirac Fermions in the Strongly-Correlated Cerium Monopnictides, arXiv:1604.08571.

[105] L. Lu, C. Fang, L. Fu, S. G. Johnson, J. D. Joannopoulos, and M. Soljacic, Symmetry-Protected Topological Photonic Crystal in Three Dimensions, Nat. Phys. 12, 337 (2016).

[106] C.-K. Chiu and A.P. Schnyder, Classification of Reflection-Symmetry-Protected Topological Semimetals and Nodal Superconductors, Phys. Rev. B 90, 205136 (2014). 
[107] S. A. Parameswaran, Topological Luttinger Invariants Protected by Crystal Symmetry in Semimetals, arXiv: 1508.01546.

[108] S. A. Parameswaran, A. M. Turner, D. P. Arovas, and A. Vishwanath, Topological Order and Absence of Band Insulators at Integer Filling in Non-Symmorphic Crystals, Nat. Phys. 9, 299 (2013).

[109] R. Roy, Space Group Symmetries and Low Lying Excitations of Many-Body Systems at Integer Fillings, arXiv: 1212.2944.

[110] H. Watanabe, H. C. Po, A. Vishwanath, and M. Zaletel, Filling Constraints for Spin-Orbit Coupled Insulators in Symmorphic and Nonsymmorphic Crystals, Proc. Natl. Acad. Sci. U.S.A. 112, 14551 (2015).
[111] P. Lowdin, On the Nonorthogonality Problem Connected with the Use of Atomic Wave Functions in the Theory of Molecules and Crystals, J. Chem. Phys. 18, 365 (1950).

[112] L. Fu and C. L. Kane, Topological Insulators with Inversion Symmetry, Phys. Rev. B 76, 045302 (2007).

[113] R. L. Dubs, S. N. Dixit, and V. McKoy, Circular Dichroism in Photoelectron Angular Distributions from Adsorbed Atoms, Phys. Rev. B 32, 8389 (1985).

[114] V. B. Zabolotnyy et al., Disentangling Surface and Bulk Photoemission Using Circularly Polarized Light, Phys. Rev. B 76, 024502 (2007).

[115] P. Giannozzi et al., QUANTUM ESPRESSO: A Modular and Open-Source Software Project for Quantum Simulations of Materials, J. Phys. Condens. Matter 21, 395502 (2009). 